\title{
Optimal operation of smart multi-energy hub systems incorporating energy hub coordination and demand response strategy
}

\author{
O. Dzobo ${ }^{\mathrm{a}, *}, \mathrm{X} \cdot \mathrm{Xia}^{\mathrm{a}}$ \\ ${ }^{a}$ Center for New Energy Systems, Department of Electrical, Electronic and Computer Engineering, University of Pretoria, \\ South Africa
}

\begin{abstract}
This paper considers the operation and optimization of a smart multi-energy hub system network using the energy hub concept. The case study system network includes integrated solar photovoltaic, thermal power generation units and natural gas CHP unit systems. A demand response - dynamic economic emission optimisation model is applied in the case study and allows for a comparison of energy hub control strategies, including the evaluation of economic, environmental criteria and power import between energy hubs. Results show a significant reduction of more than $50 \%$ in both total generation cost and amount of emission when different energy hub control strategies are employed. The results also show that load shifting capabilities of different energy hub loads cannot be ignored as it reduces the electricity bill of energy hub customers.

Keywords: demand response, economic load dispatch, emission dispatch, transmission power loss
\end{abstract}

\section{Introduction}

The continuous growth in energy demand, dependency on fossil fuels, integration of distributed generation units and the increasing societal desire to utilize more sustainable and environmentally friendly energy sources represent future challenges for both energy system planning and operation [1, 2]. Many researchers have been 5 re-oriented to model this new decision making environment and to propose new power system management frameworks in order to optimally control the interaction between these aspects [3. Several conceptual approaches have been examined for describing energy systems with a mix of energy sources [4. The 'energy hub concept', has been initiated at ETH Zurich within the project "Vision of Future Energy Networks" to address these kind of energy systems [5]. Recent research studies have started to address the integrated control of energy hub systems [6]. However, an increase in the number of geographically dispersed energy hub systems connected to the power system network is expected in the near future. From this perspective, it is crucial to develop reliable and cost effective operational models of the interconnected energy hub systems to properly dispatch their input energy carriers, which could be characterized by different constraints.

The expansion of natural gas networks and its use in Combined Heat and Power (CHP) technologies have gained high level of popularity as one of the most important distributed energy resources in many developed

\footnotetext{
${ }^{*}$ Corresponding author

Email addresses: odzobo@yahoo.com (O. Dzobo), xxia@up.ac.za (X. Xia)
} 
countries 4, 1, 8. Recently, the discovery of natural gas in Mozambique, 9, has led the South African government to propose the construction of a natural gas pipeline network between the two countries [10]. The use of natural gas in CHP technologies to simultaneously generate heat and power in some industrial, large institutional facilities or other commercial facilities in South Africa is therefore expected to increase in the near future. Hence, in this paper a typical energy hub system network with three energy hubs containing CHP generation units and solar photovoltaic (PV) power generation units is used as a case study.

A relevant number of recent research studies have dealt with characterisation and optimization of decentralised multi-energy systems [6, 4, 11, 12. The characterisation and optimization mathematical modelling focuses mainly on providing optimal choice of energy hub components, interaction between energy hubs and other energy infrastructures; and optimal energy carriers purchase and storage utilization. As more decentralised energy hub systems become geographically dispersed, the coordination of these energy hubs have become very important. The coordination between the energy hubs can be categorized as centralized and distributed [13. In a centralized coordination of energy hubs, a central supervisory controller measure all parameters in the system network and solves at each decision step, one optimization problem under the measured parameters constraints to determine actions for the entire system. Figure 1 a shows the centralized control architecture where a central supervisory controller supervises three interconnected energy hubs. Distributed coordination of energy hubs is achieved when each energy hub has its own respective distributed
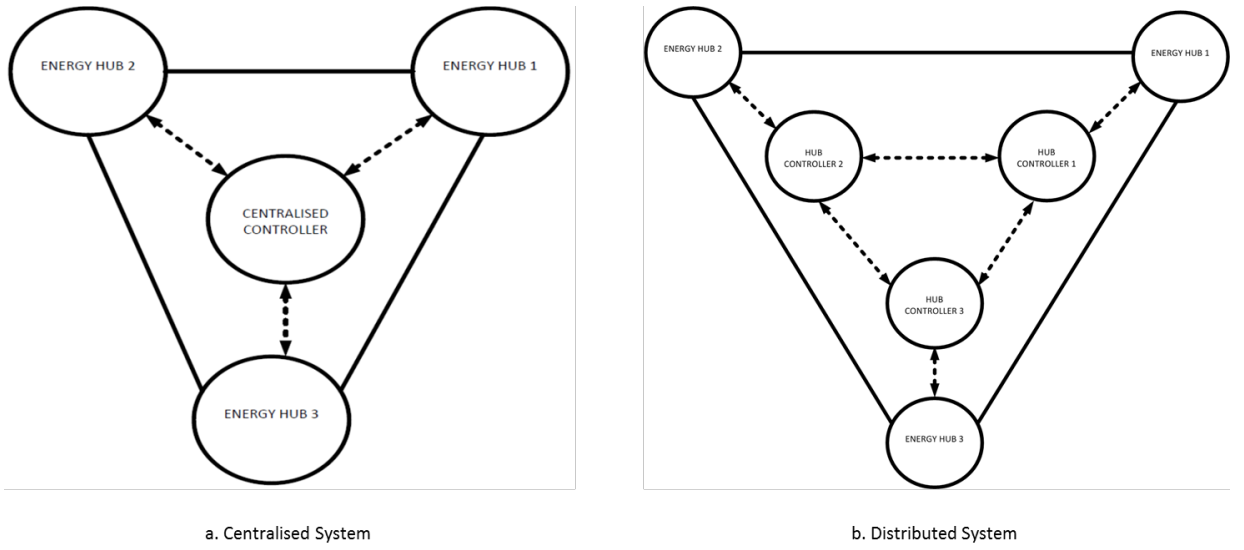

Figure 1: Different control architectures for interconnected energy hubs

supervisory controller as shown in Figure 1.b. The overall optimization problem is divided into subproblems among the respective distributed supervisory controllers and solved in an iterative procedure. Each distributed supervisory controller has to coordinate its actions among neighbouring energy hubs in order to optimize the entire system network and guarantee continuous energy supply.

In most research studies in the literature, the optimization mathematical models of energy hubs is separated from the energy hub load [14, 15, 16, 17. These optimization models include both deterministic and stochastic models [18. More complex multi-energy hub systems optimization models also appear in the literature, where deterministic model predictive control approaches are used for the optimal operation of the energy hubs [13, 18. The main disadvantage of decoupling the operation of the energy hub and the interconnected load, is the underutilization of the load shifting capabilities of the load in the interconnected 
system. This may lead to suboptimal operation of the energy systems present in the energy hub. Several other authors have addressed demand response strategies combined with energy hub system optimisation in short-term and multi-period optimization models. Ref.[19] introduce the energy hub concept with stochastic demand response using model predictive control (MPC). The demand response load model for the connected energy hub customers is lumped together and is taken as a stochastic variable. Centralised and distributed control schemes of the energy hub systems were considered in the analyses. In ref. [20, the mathematical optimization formulation described has all the input energy hub load data specified at fixed times using deterministic values. These analyses however, have the disadvantage of that different energy hub loads operate at different times during the day and it is difficult to see the actual allocation of time for the individual energy hub loads. In some cases, the energy hub loads are power shiftable and/or sequentially time shiftable which may not be taken into account in the analysis. Therefore, the use of stochastic optimal energy hub loads or non-shftable deterministic energy hub loads might not reflect the best times of operation for the individual energy hub loads. In this paper, individual energy hub loads for three different energy hub customers are considered. In addition, sequential time-shiftable and power shiftable energy hub loads are considered in the analyses. A combined demand response - dynamic economic emission dispatch (DR-DEED) strategy for future power system networks in the context of multi-energy hub systems is presented. Both centralised and distributed control strategies are used for coordination of the energy hubs. The objective of the combined DR-DEED strategy is to maximize the economic benefit of both electricity customers and power utility through reducing their electricity bill/cost, and reducing the generation cost and amount of emission of the thermal power generation units.

The contribution of the current research paper can be summarized as follows:

- A multi-energy system network with three energy hubs is considered as a case study. Each energy hub has a mix of energy systems, i.e., thermal power generation units, CHP units and solar PV power generation units.

- The demand response program is proposed in order to have a more successful participation of CHP units and solar PV power generation units in the power market. In the proposed DR program, sequential time-shiftable and power shiftable loads are considered.

- Non-convex feasible operation region in different types of CHP units is modelled as a mixed-integer linear formulation. In addition, the ramp rates of both thermal power generation units and CHP units are also considered in the analysis.

- Two control strategies are used for the coordination of the energy hubs, i.e., centralised and distributed control strategies.

\section{Energy Hub Concept}

An example of an energy hub is presented in Figure 2. The energy hub output port provides electricity, heating, and cooling to the energy hub customer loads. Both converters and storage devices are incorporated 


\section{ENERGY HUB}

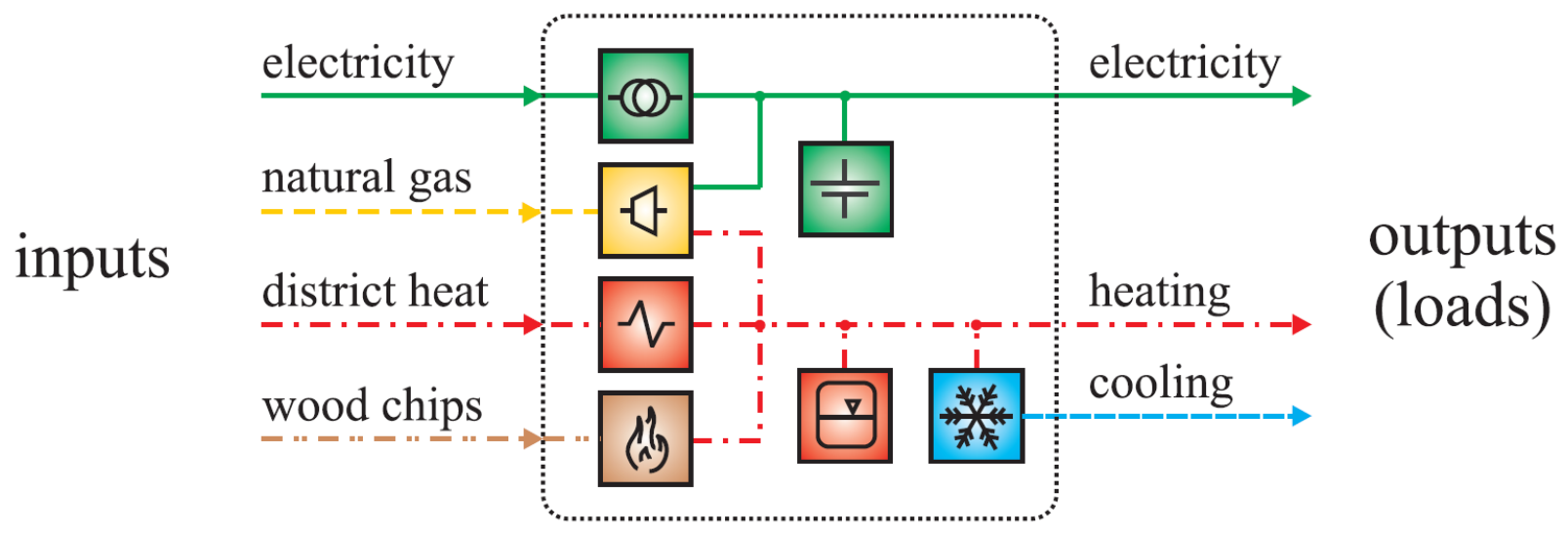

Figure 2: Example of an energy hub

in the energy hub system. The converters, e.g., electric transformer, CHP device, furnace are used to change input carrier energy into another form of energy. In some cases, direct connections between the energy hub input and output ports are used when there is no change in the input energy. For example, electric cables and/or overhead lines can be used to transport electricity to the energy hub output port. For storage of excess energy like power or heat, storage devices are incorporated within the energy hub system. For example, batteries are used for storage of electric energy and/or steam boilers for conserving heat energy. Figure 3 a shows a converter with one input and one output, such as a transformer. At any given time, $t$,

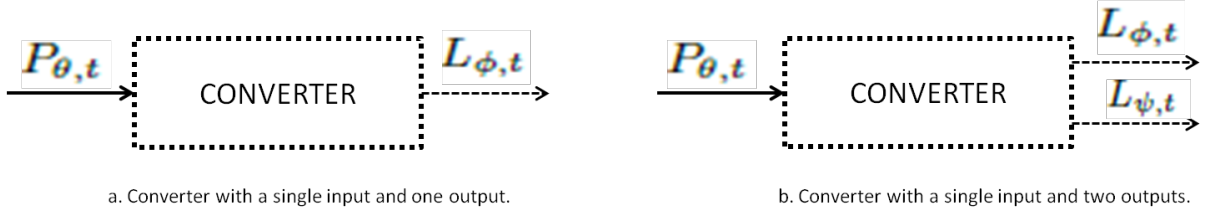

Figure 3: Model of power converters with inputs and outputs

the input power $P_{\theta, t}$ and output power $L_{\phi, t}$ are coupled as:

$$
L_{\phi, t}=C_{\theta \phi} * P_{\theta, t}
$$

where $C_{\theta \phi}$ is the converter efficiency for the power conversion of the device. Similarly, Figure 3 . b can be expressed as:

$$
\left[\begin{array}{c}
L_{\phi, t} \\
L_{\theta, t}
\end{array}\right]=\left[\begin{array}{c}
C_{\theta \phi} \\
C_{\theta \theta}
\end{array}\right] P_{\theta, t}
$$

In this case, $C_{\theta \phi}$ and $C_{\theta \theta}$ are the coupling factors correspond to the converter's energy efficiencies. When 90 various energy carriers and converter elements are included in the energy hub, it leads to a general formulation 
of the multi-input multi-output (MIMO) energy hub system as shown in Eq3 below.

$$
\left[\begin{array}{c}
L 1_{\phi, t} \\
L 2_{\theta, t} \\
\cdot \\
\cdot \\
\cdot \\
L n_{\omega, t}
\end{array}\right]=\left[\begin{array}{cccccc}
C 1_{\phi \phi} & C 1_{\theta \phi} & . & \cdot & . & C 1_{\omega \phi} \\
C 2_{\phi \theta} & C 2_{\theta \theta} & . & . & \cdot & C 2_{\omega \theta} \\
\cdot & \cdot & \cdot & \cdot & \cdot & \cdot \\
\cdot & \cdot & \cdot & \cdot & \cdot & \cdot \\
\cdot & \cdot & . & \cdot & \cdot & \cdot \\
C n_{\phi \omega} & C n_{\theta \omega} & \cdot & \cdot & \cdot & C n_{\omega \omega}
\end{array}\right]\left[\begin{array}{c}
P_{\phi, t} \\
P_{\theta, t} \\
\cdot \\
\cdot \\
\cdot \\
P_{\omega, t}
\end{array}\right]
$$

\section{CASE STUDY}

Figure 4 shows the integrated energy hub system network interconnected by natural gas and electricity networks. The electricity network comprises three network nodes, whereas the natural gas network only features two network nodes. This case study represents a typical urban energy supply network that is divided into residential load (Hub 1), commercial load (Hub 2) and industrial load (Hub 3) supply areas. The internal structure of each energy hub depends on the specific loads present at that energy hub. Each energy hub has

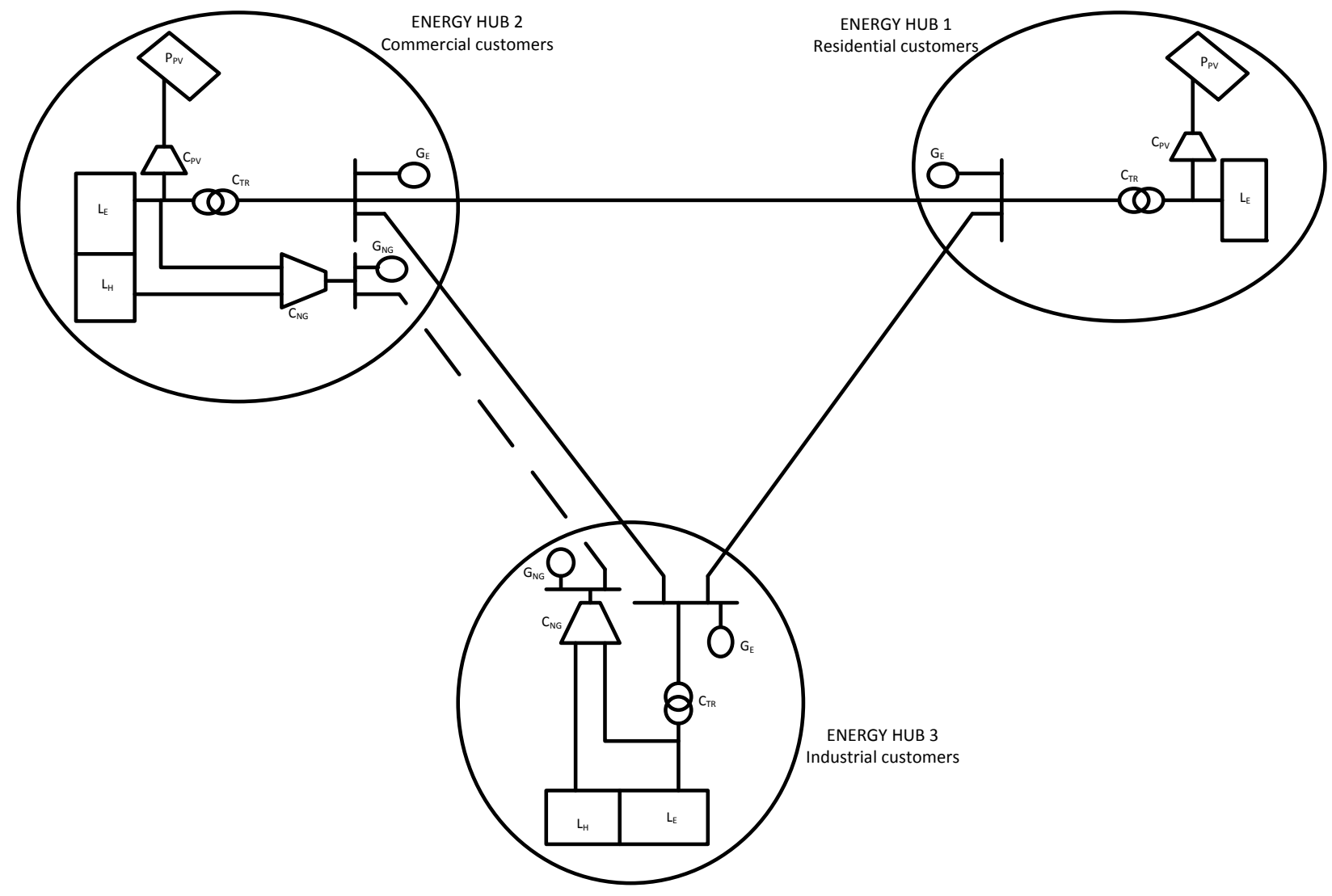

Figure 4: Single-line diagram of the smart multi-energy hub system network

its own local electrical energy production $G_{p v i}$ and $G_{e i}$, and heat production $G_{n g i}$ for $i \in 1,2, \ldots, n$. Energy hubs 2 and 3 consume electric power $G_{e i}$ and natural gas $G_{n g i}$, and supplies energy to its electric load $L_{e k}$ 
and heat load $L_{h k}$ for $k \in 1,2, \ldots, K$. Energy hub 1 has only electric load $L_{e k}$ and is supplied by solar PV power generation $G_{p v i}$ and thermal power generation $G_{e i}$. Tables 1 - 2 show the converter efficiency data and; thermal power generation and CHP generation unit data respectively, for the multi-energy hub system network. The TOU electricity prices and heat demand for the energy hub customers are presented in Tables 3-4 respectively. Aggregate energy hub loads for residential, commercial and industrial customers connected at the different energy hubs are presented in Tables 5,6 and 7 respectively.

Table 1: Converter efficiency data of the multi-energy hub system network

\begin{tabular}{lllllll}
\hline$C_{P V 1}$ & $C_{P V 2}$ & $C_{T R 1}$ & $C_{T R 2}$ & $C_{T R 3}$ & $C_{N G 2}$ & $C_{N G 3}$ \\
\hline 0.95 & 0.95 & 0.98 & 0.98 & 0.98 & 0.96 & 0.96 \\
\hline
\end{tabular}

Table 2: Thermal generation unit data of the multi-energy hub system network

\begin{tabular}{|c|c|c|c|c|c|c|c|c|c|c|c|}
\hline $\begin{array}{l}\text { Hub } \\
\text { No. }\end{array}$ & $\begin{array}{l}\text { Gen } \\
\text { No. }\end{array}$ & $\begin{array}{l}P_{i}^{\max } \\
\text { MW }\end{array}$ & $\begin{array}{l}P_{i}^{\min } \\
M W\end{array}$ & $\begin{array}{l}a_{i} \\
\$ / \mathrm{h}\end{array}$ & $\begin{array}{l}b_{i} \\
\$ / \mathrm{MWh}\end{array}$ & $\begin{array}{l}c_{i} \\
\$ / \mathrm{MW}^{2} \mathrm{~h}\end{array}$ & $\begin{array}{l}d_{i} \\
\mathrm{lb} / \mathrm{h}\end{array}$ & $\begin{array}{l}e_{i} \\
\mathrm{lb} / \mathrm{MWh}\end{array}$ & $\begin{array}{l}f_{i} \\
\mathrm{lb} / \mathrm{MW}^{2} \mathrm{~h}\end{array}$ & $\begin{array}{l}U R_{i} \\
\mathrm{MW} / \mathrm{h}\end{array}$ & $\begin{array}{l}D R_{i} \\
\mathrm{MW} / \mathrm{h}\end{array}$ \\
\hline \multirow{3}{*}{ Hub 1} & 1 & 500 & 100 & 200 & 38.5397 & 0.007 & 13.8593 & 0.32767 & 0.00419 & 80 & 120 \\
\hline & 2 & 200 & 50 & 1450.7045 & 38.2704 & 0.0095 & 330.0056 & 0.32767 & 0.00419 & 50 & 90 \\
\hline & 3 & 300 & 80 & 1450.7045 & 38.3055 & 0.009 & 330.0056 & -0.54551 & 0.00683 & 65 & 100 \\
\hline \multirow{5}{*}{ Hub 2} & 1 & 340 & 73 & 1469.4026 & 40.3965 & 0.03280 & 360.0012 & 0.32767 & 0.00419 & 80 & 80 \\
\hline & 2 & 300 & 63 & 200 & 11.0 & 0.00354 & 38.3055 & -0.51116 & 0.00461 & 50 & 50 \\
\hline & 3 & 130 & 20 & 220 & 36.5104 & 0.0121 & 42.8955 & -0.51116 & 0.00461 & 30 & 30 \\
\hline & 4 & 55 & 10 & 200 & 40.5407 & 00.1295 & 40.2669 & -0.51116 & 0.00683 & 30 & 30 \\
\hline & $C H P 1$ & 247 & 98.8 & 220 & 10.5 & 0.00345 & - & - & - & 70 & 70 \\
\hline \multirow{5}{*}{ Hub 3} & 1 & 500 & 100 & 190 & 38.5397 & 0.007 & 42.8955 & 0.32767 & 0.00419 & 80 & 120 \\
\hline & 2 & 200 & 50 & 1455.6056 & 38.2704 & 0.0095 & 350.0056 & 0.32767 & 0.00419 & 50 & 90 \\
\hline & 3 & 470 & 135 & 220 & 38.5397 & 0.009 & 40.2669 & -0.54551 & 0.00683 & 80 & 80 \\
\hline & 4 & 160 & 57 & 240 & 39.5804 & 0.0179 & 13.8593 & -0.51116 & 0.00461 & 50 & 50 \\
\hline & $C H P 2$ & 125.8 & 45.1 & 240 & - & 0.0265 & 13.8593 & - & - & 50 & 50 \\
\hline
\end{tabular}

Table 3: TOU electricity prices of the multi-energy hub system network for 24 hours.

\begin{tabular}{|l|l|l|l|l|l|l|l|l|l|l|l|l|}
\hline Time $(t)$ & 1 & 2 & 3 & 4 & 5 & 6 & 7 & 8 & 9 & 10 & 11 & 12 \\
\hline Residential TOU price & 0.4088 & 0.4088 & 0.4088 & 0.4088 & 0.4088 & 0.6413 & 0.6413 & 0.9293 & 0.9293 & 0.9293 & 0.6413 & 0.6413 \\
\hline Commercial TOU price & 0.3979 & 0.3979 & 0.3979 & 0.3979 & 0.3979 & 0.6272 & 0.6272 & 0.9112 & 0.9112 & 0.9112 & 0.6272 & 0.6272 \\
\hline Industrial TOU price & 0.3853 & 0.3853 & 0.3853 & 0.3853 & 0.3853 & 0.6073 & 0.6073 & 0.8825 & 0.8825 & 0.8825 & 0.6073 & 0.6073 \\
\hline \hline Time $(t)$ & 13 & 14 & 15 & 16 & 17 & 18 & 19 & 20 & 21 & 22 & 23 & 24 \\
\hline Reidential TOU price & 0.6413 & 0.6413 & 0.6413 & 0.6413 & 0.6413 & 0.6413 & 0.9293 & 0.9293 & 0.6413 & 0.6413 & 0.4088 & 0.4088 \\
\hline Commercial TOU price & 0.6272 & 0.6272 & 0.6272 & 0.6272 & 0.6272 & 0.6272 & 0.9112 & 0.9112 & 0.6272 & 0.6272 & 0.3979 & 0.3979 \\
\hline Industrial TOU price & 0.6073 & 0.6073 & 0.6073 & 0.6073 & 0.6073 & 0.6073 & 0.8825 & 0.8825 & 0.6073 & 0.6073 & 0.3853 & 0.3853 \\
\hline
\end{tabular}

\subsection{Energy Hub system components modelling}

\subsubsection{Combined Heat and Power units modelling}

The heat and power outputs of CHP units are interdependent. In this case study, two type of feasible operation regions (FOR) for CHP units are considered. The first type of CHP unit is shown in Fig 5 . The FOR of the CHP unit shown is constrained by three operational parameters: maximum power generation, minimum power generation and maximum heat generation. The FOR of the CHP unit is defined by the boundary curve $\mathrm{ABCD}$. In the graph, it can be seen that the heat generation increases as the power generation 
Table 4: Heat demand of the different energy hubs for 24 hours.

\begin{tabular}{|l|l|l|l|l|l|l|l|l|l|l|l|l|l|l|l|l|l|l|l|l|l|l|l|l|}
\hline Time $(t)$ & 1 & 2 & 3 & 4 & 5 & 6 & 7 & 8 & 9 & 10 & 11 & 12 & 13 & 14 & 15 & 16 & 17 & 18 & 19 & 20 & 21 & 22 & 23 & 24 \\
\hline Hub 2 & 165 & 152 & 163 & 140 & 145 & 173 & 109 & 133 & 136 & 160 & 111 & 145 & 190 & 151 & 163 & 150 & 121 & 121 & 69 & 132 & 123 & 84 & 75 & 100 \\
\hline Hub 3 & 125 & 102 & 103 & 130 & 135 & 63 & 89 & 102 & 112 & 115 & 120 & 123 & 119 & 125 & 126 & 125 & 122 & 120 & 115 & 109 & 102 & 84 & 75 & 60 \\
\hline
\end{tabular}

Table 5: Aggregate residential customer loads

\begin{tabular}{lllll}
\hline Type of load & $E_{D A I L Y}(\mathrm{MWh})$ & $\alpha_{a}(\mathrm{~h})$ & $\beta_{a}(\mathrm{~h})$ & $Z_{a}(\mathrm{~h})$ \\
\hline Inflexible & & & & \\
Load 1 & 300 & 1 & 24 & 24 \\
Load 2 & 100 & 12 & 16 & 5 \\
& & 20 & 22 & 3 \\
Flexible & & & & \\
Load 3 & Hourly consumption: $0-50$ & 1 & 24 & - \\
& Daily requirement: 200 & & & \\
Load 4 & Hourly consumption: 0 - 150 & 21 & 9 & - \\
& Daily requirement: 550 & & & \\
Load 5 & Hourly consumption: $0-100$ & 20 & 8 & - \\
& Daily requirement: 400 & & & \\
Load 6 & 50 & 8 & 20 & 3 \\
Load 7 & 150 & 16 & 22 & 4 \\
\hline
\end{tabular}

Table 6: Aggregate commercial customer loads

\begin{tabular}{lllll}
\hline Type of load & $E_{D A I L Y}(\mathrm{MWh})$ & $\alpha_{a}(\mathrm{~h})$ & $\beta_{a}(\mathrm{~h})$ & $Z_{a}(\mathrm{~h})$ \\
\hline Inflexible & & & & \\
Load 1 & 400 & 1 & 24 & 24 \\
Load 2 & 80 & 12 & 16 & 3 \\
& 80 & 15 & 17 & 3 \\
Load 3 & 110 & 10 & 14 & 5 \\
& 50 & 16 & 20 & 5 \\
Load 4 & 50 & 8 & 8 & 1 \\
& 150 & 12 & 12 & 1 \\
Flexible & Hourly consumption: $0-300$ & 8 & 20 & - \\
Load 5 & Daily requirement: 800 & & & \\
& Hourly consumption: $20-50$ & 01 & 6 & - \\
Load 6 & $: 50-100$ & 21 & 24 & - \\
& Daily requirement: 350 & & & \\
Load 7 & 50 & 8 & 20 & 3 \\
Night-time & & & & \\
Load 8 & 150 & 21 & 03 & 4 \\
\hline
\end{tabular}


Table 7: Aggregate industrial customer loads

\begin{tabular}{|c|c|c|c|c|}
\hline Type of load & $E_{D A I L Y}(\mathrm{MWh})$ & $\alpha_{a}(\mathrm{~h})$ & $\beta_{a}(\mathrm{~h})$ & $Z_{a}(\mathrm{~h})$ \\
\hline \multicolumn{5}{|l|}{ Inflexible } \\
\hline Load 1 & 500 & 1 & 24 & 24 \\
\hline \multirow[t]{2}{*}{ Load 2} & 100 & 10 & 17 & 8 \\
\hline & 70 & 18 & 20 & 3 \\
\hline \multicolumn{5}{|l|}{ Flexible } \\
\hline \multirow[t]{2}{*}{ Load 3} & Hourly consumption: $50-100$ & 7 & 16 & - \\
\hline & Daily requirement: 300 & & & \\
\hline Load 4 & 150 & 9 & 20 & 6 \\
\hline \multicolumn{5}{|l|}{ Night-time } \\
\hline \multirow[t]{2}{*}{ Load 5} & 50 & 21 & 24 & 5 \\
\hline & & 01 & 06 & 6 \\
\hline Load 6 & 150 & 22 & 3 & 6 \\
\hline \multirow[t]{2}{*}{ Load 7} & Hourly consumption: $100-250$ & 21 & 6 & - \\
\hline & Daily requirement: 700 & & & \\
\hline \multirow[t]{2}{*}{ Load 8} & $1^{s t}$ Hour: 100 & 21 & 6 & - \\
\hline & $6^{t h}$ Hour: 50 & & & \\
\hline
\end{tabular}

decreases along the boundary curve $\mathrm{AB}$, while along the curve $\mathrm{BC}$, both power generation and heat generation decreases. The FOR of the CHP unit is defined by Eqs 4, 5, 6, 7 and 8 .

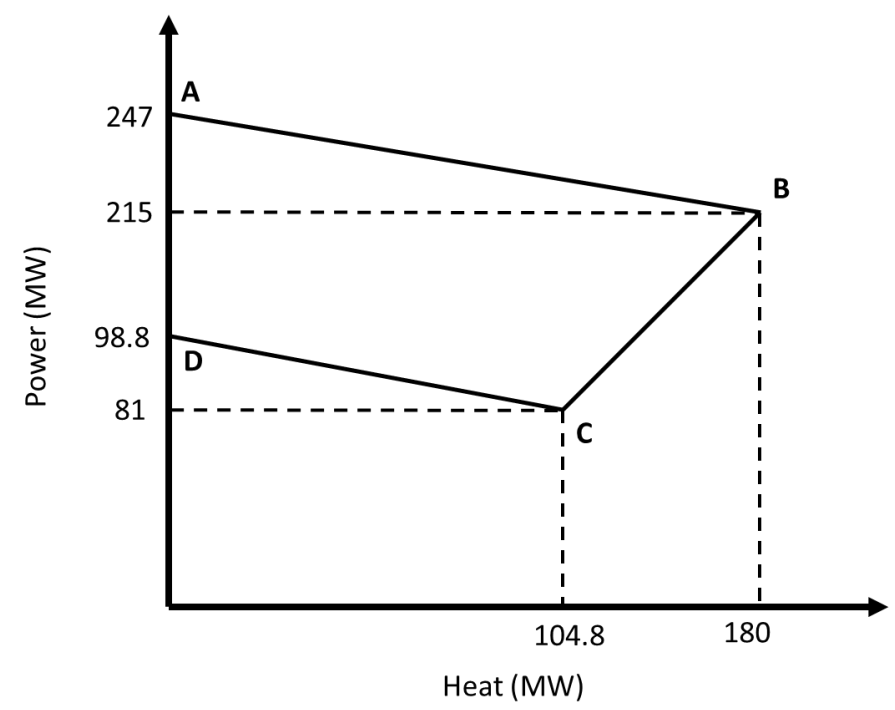

Figure 5: Heat-power feasible operation region for the CHP unit at Hub 2

$$
\begin{aligned}
P_{i, t}-P_{i, A}-\frac{P_{i, A}-P_{i, B}}{H_{i, A}-H_{i, B}}\left(H_{i, t}-H_{i, A}\right) & \leq 0 \\
\Rightarrow P_{i, t}+\frac{8}{45} H_{i, t}-247 & \leq 0 \\
P_{i, t}-P_{i, B}-\frac{P_{i, B}-P_{i, C}}{H_{i, B}-H_{i, C}}\left(H_{i, t}-H_{i, B}\right) & \leq 0 \\
\Rightarrow P_{i, t}-\frac{134}{75.2} H_{i, t}+105.74468 & \leq 0 \\
P_{i, t}-P_{i, C}-\frac{P_{i, C}-P_{i, D}}{H_{i, C}-H_{i, D}}\left(H_{i, t}-H_{i, C}\right) & \leq 0
\end{aligned}
$$




$$
\begin{aligned}
& \Rightarrow P_{i, t}+\frac{17.8}{104.8} H_{i, t}-98.8 \leq 0 \\
& 0 \leq H_{i, t} \leq 180 \\
& 0 \leq P_{i, t} \leq 247
\end{aligned}
$$

where indices A, B, C and D represent four parameter value points that borders the FOR of the CHP unit. Eq. 4 defines the area under the curve $\mathrm{AB}$ and Eq. 5 represents the area above the curve BC. The area above curve CD is defined using Eq. 6. According to Eqs. 7] and 8, the heat and power generation output of the CHP unit is set to zero if it is not operational and during operation the CHP unit is restricted above by the maximum heat generation and power generation respectively. The CHP unit has ramp rates during start and shut down times of its operation. Eq. 9 presents a constraint to ensures that the CHP unit ramp rate limits are not violated.

$$
-D R_{i}^{C H P} \leq P_{i, t+1}^{C H P}-P_{i, t}^{C H P} \leq U R_{i}^{C H P}
$$

where $D R_{i}^{C H P}$ and $U R_{i}^{C H P}$ are maximum ramp down rates and maximum ramp up rates of the $i^{\text {th }} \mathrm{CHP}$ unit, respectively.

The second type of CHP unit is shown in Fig 6. The FOR of this CHP unit is defined by the boundary curve ABCDE. The heat generation increases as the power generation decreases along boundary BC, while both heat and power generation decreases along the curve CD. Similarly, the second type CHP unit is constrained by the same three operational parameters as the first type CHP unit. The FOR of this CHP unit is defined by Eqs. 10, 11, 12, 13 and 14 .

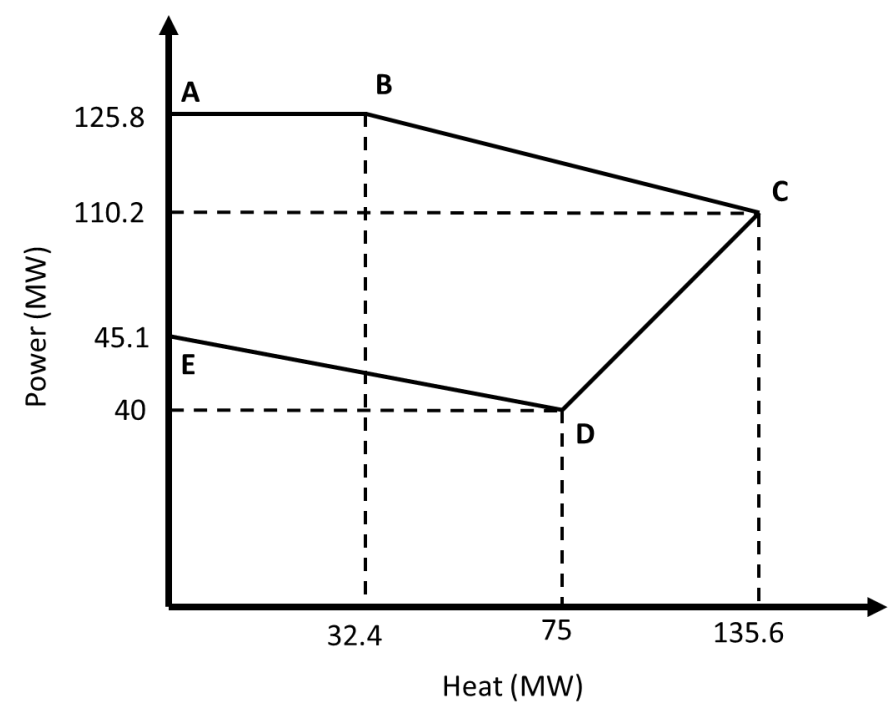

Figure 6: Heat-power feasible operation region for the CHP unit at Hub 3

$$
P_{i, t}-P_{i, B}-\frac{P_{i, B}-P_{i, C}}{H_{i, B}-H_{i, C}}\left(H_{i, t}-H_{i, B}\right) \leq 0
$$




$$
\begin{array}{r}
\Rightarrow P_{i, t}-\frac{15.6}{103.2} H_{i, t}-130.69767 \leq 0 \\
P_{i, t}-P_{i, C}-\frac{P_{i, C}-P_{i, D}}{H_{i, C}-H_{i, D}}\left(H_{i, t}-H_{i, C}\right) \leq 0 \\
\Rightarrow P_{i, t}-\frac{5.1}{75} H_{i, t}-45.1 \leq 0 \\
P_{i, t}-P_{i, D}-\frac{P_{i, D}-P_{i, E}}{H_{i, D}-H_{i, E}}\left(H_{i, t}-H_{i, D}\right) \leq 0 \\
\Rightarrow P_{i, t}-\frac{70.2}{60.6} H_{i, t}-46.881188 \leq 0 \\
0 \leq H_{i, t} \leq 135.6 \\
0 \leq P_{i, t} \leq 125.8
\end{array}
$$

where indices A, B, C, D and E represents the five parameter value points that borders the FOR of the CHP

unit. Eq. 10 describes the area under the curve $\mathrm{BC}$ and the area above the curve $\mathrm{DE}$ is defined by using Eq. 12. Eq. 11 defines the area above CD. When the CHP unit is not operational, the heat and power generation output of the CHP unit is set to zero and during operation the CHP unit is constrained by its maximum heat and power generation values according to Eqs. 13 and 14 . The CHP unit has ramp rates during start and shut down times of its operation. Eq. 15 presents a constraint to ensures that the CHP unit ramp rate limits are not violated.

$$
-D R_{i} \leq P_{i, t+1}-P_{i, t} \leq U R_{i}
$$

where $D R_{i}$ and $U R_{i}$ are maximum ramp down rates and maximum ramp up rates of the $i^{\text {th }}$ CHP unit, respectively.

In this paper, a CHP unit is modelled to have a convex cost function in both power and heat generation 21. The form of the fuel cost function of CHP units adopted in this paper is given as follows:

$$
C_{i}^{C H P}\left(P_{i, t}^{C H P}, H_{i, t}^{C H P}\right)=a_{i}+b_{i} P_{i, t}^{C H P}+c_{i}\left(P_{i, t}^{C H P}\right)^{2}+d_{i} H_{i, t}^{C H P}+e_{i}\left(H_{i, t}^{C H P}\right)^{2}+f_{i}\left(P_{i, t}^{C H P}, H_{i, t}^{C H P}\right)
$$

\subsubsection{Convectional Thermal Power generation units modelling}

The generation cost function of thermal power generation units have been derived using linear cost function, piecewise linear cost function, quadratic cost function etc [22]. In this paper, the quadratic generation cost function is adopted. The quadratic generation cost function is the most commonly adopted function in the literature and it is said to be a more accurate function than others. The generation cost function for thermal power generation units is defined in terms of its output power. The generation cost function can be expressed as follows:

$$
C_{i}^{G}\left(P_{i, t}\right)=a_{i}+b_{i} P_{i, t}^{G}+c_{i}\left(P_{i, t}^{G}\right)^{2}
$$

As thermal power generation units burnt the fuel for their power output there are a lot of emission as a result of such operation. The emission function of the thermal power generation units is expressed in terms of the sum of all types of emissions, common examples are $\mathrm{NO}, \mathrm{SO}_{2}$ and $\mathrm{CO}_{2}$. In this paper, the emission functions for $\mathrm{NO}, \mathrm{SO}_{2}$ and $\mathrm{CO}_{2}$ are taken as quadratic functions and are expressed as follows: 


$$
E_{i}\left(P_{i, t}\right)=g_{i}+h_{i} P_{i, t}+m_{i} P_{i, t}^{2}
$$

The thermal power generation unit operation constraints ensures that the thermal power generation unit's power output limits are not exceeded, i.e,

$$
P_{i}^{\min } \leq P_{i} \leq P_{i}^{\max }
$$

where $P_{i}^{\text {min }}$ and $P_{i}^{\max }$ are lower and upper bounds for power outputs of the $i^{\text {th }}$ thermal power generation unit, respectively. The thermal power generation units have ramp rates during start and shut down times

of its operation. Eq. 20 presents a constraint to ensures that the thermal power generation unit ramp rate limits are not violated.

$$
-D R_{i} \leq P_{i, t+1}-P_{i, t} \leq U R_{i}
$$

where $D R_{i}$ and $U R_{i}$ are maximum ramp down rates and maximum ramp up rates of the $i^{\text {th }}$ thermal power generation unit, respectively.

\subsubsection{Demand Response load modelling}

The energy hub customers are assumed to have the same behaviour in terms of the varying load response to electricity price variation. The electricity price information is released a day ahead and the energy hub supervisory controller arrange the operation of energy hub load for the next 24 hours. The scheduling horizon and the resolution of the scheduling horizon are assumed to be 24 hours and one hour respectively. The DR objective function minimizes the electricity bill of the energy hub customers without affecting their daily energy requirements. Let $\mathbf{A}$ denote the set of energy hub load demand, then: $L_{a}^{t} \geq 0$ is the load demand by energy hub load $a \in \mathbf{A}$ at time $t$. The total daily energy requirement from energy hub load $a$ over one day can be defined as:

$$
\mathbf{E}_{\mathbf{D A I L Y}, \mathbf{a}}=\sum_{t=1}^{T} L_{a}^{t}
$$

For inflexible energy hub load which cannot be shifted to any time slot, the total energy requirement for the whole operation period of the energy hub load is given by:

$$
\sum_{t=\alpha_{a}}^{\beta_{a}} L_{a}^{t}=E_{a} \quad \forall a \in \mathbf{A}
$$

where $E_{a}$ is the total energy requirement for the whole operation of energy hub load, $\alpha_{a}$ is the beginning of acceptable operation time and $\beta_{a}$ is the end of acceptable operation time. Eq. 22 ensures that the operation period of the energy hub load is finished before deadline and is equal to the total energy requirement of operation. It is also required that $L_{a}^{t}=0 \quad \forall t<\alpha_{a}$ and $t>\beta_{a}$. For example, Load 2 in Table 5 it has two periods of operation, i.e., $\left(\alpha_{1}=12, \beta_{1}=16\right)$ and $\left(\alpha_{2}=20, \beta_{2}=22\right)$. The total daily energy requirement for Load 2 is given as:

$$
\begin{aligned}
E_{D A I L Y, \text { Load } 2} & =\sum_{t=12}^{16} L_{\text {Load } 2}^{t}+\sum_{t=20}^{22} L_{\text {Load } 2}^{t} \\
& =500+300 \\
& =800 M W h
\end{aligned}
$$


It is also required that $L_{\text {Load } 2}^{t}=0 \forall 1 \leq t \leq 11,17 \leq t \leq 19$ and $23 \leq t \leq 24$.

In case where the hourly energy hub load demand is known in terms of range of load levels, then $L_{a}^{t}$ is treated as variable. For energy hub load $a \in \mathbf{A}$ which have a maximum hourly load level $\gamma_{a}^{\max }$ and a minimum hourly load level $\gamma_{a}^{\text {min }}$, the total daily energy requirement is given as:

$$
\sum_{t=\alpha_{a}}^{\beta_{a}} L_{a}^{t}=E_{D A I L Y, a} \quad \forall a \in \mathbf{A} \quad t \in\left[\alpha_{a}, \beta_{a}\right], \gamma_{a}^{\min } \leq L_{a}^{t} \leq \gamma_{a}^{\max }
$$

180

For example, Load 3 in Table 5 , the energy hub load operates for the whole 24 hours of the day, i.e., $\alpha=1, \beta=24$. The energy hub load can take any value between $\gamma_{\text {Load } 3}^{\min }=0$ and $\gamma_{\text {Load } 3}^{\max }=50 M W h$ at each hour $t$. However, the total daily energy requirement for the energy hub load must be equal to 200MWh.

Flexible energy hub loads can be arranged in several hours while ensuring the total energy supplies. Let $\mathbf{R}(\subset \mathbf{A})$ denote the set of indexes of the flexible energy hub load. For energy hub load $a \in \mathbf{R}$, if $X_{a}$ denote the fundamental load demand pattern as $\left(\varepsilon_{a}^{1}, \varepsilon_{a}^{2}, \ldots, \varepsilon_{a}^{t}, \ldots, \varepsilon_{a}^{T}\right)$ where $\varepsilon_{a}^{t} \geq 0$, the $a^{\text {th }}$ flexible energy hub load can have $\mathrm{T}$ possible pattens which are obtained by circular shifting the fundamental load demand pattern. In order to select one possible load demand pattern for optimization, a binary switching integer vector $s_{a}$ is used. The binary switching integer vector $s_{a}$ is defined as $s_{a}=\left(s_{a}^{1}, s_{a}^{2}, \ldots, s_{a}^{t}, \ldots, s_{a}^{T}\right)$ where $s_{a}^{t} \in(0,1)$. The position of a binary integer one (1) means the starting time at which the energy hub load is switched $\mathrm{ON}$. The binary switching integer vector therefore has only one non-zero element equal to one (1) in order to ensure that each energy hub load is switched only once per each operation. For energy hub load $a \in \mathbf{R}$, this constraint can be written as :

$$
\sum_{t=1}^{T} s_{a}^{t}=1
$$

By using $s_{a}$, the energy hub load demand scheduling plan $L_{a}$ can be written as:

$$
L_{a}=s_{a} * \mathrm{X}_{a}^{\mathbf{C}} \quad \forall a \in \mathbf{R}
$$

where the columns of the $24 \times 24$ matrix $X_{a}^{C}$ is the circulant matrix of the fundamental load demand pattern, $X_{a}$, i.e.

$$
\mathrm{X}_{a}^{C}=\left(\begin{array}{ccccccc}
\varepsilon_{a}^{1} & \varepsilon_{a}^{24} & . & . & . & . & \varepsilon_{a}^{2} \\
\varepsilon_{a}^{2} & \varepsilon_{a}^{1} & . & . & . & . & \varepsilon_{a}^{3} \\
\cdot & . & . & . & . & . & . \\
\cdot & \cdot & . & . & . & . & . \\
\cdot & . & . & . & . & . & . \\
\varepsilon_{a}^{24} & \varepsilon_{a}^{23} & . & . & . & . & \varepsilon_{a}^{1}
\end{array}\right) .
$$

For simplicity, lets take a 5 hour load schedule for energy hub load $a$. If the fundamental load demand pattern is given as $X_{a}=\left[\begin{array}{lllll}100 & 0 & 0 & 50 & 0\end{array}\right]$ then:

$$
\mathrm{X}_{a}^{C}=\left(\begin{array}{ccccc}
100 & 0 & 50 & 0 & 0 \\
0 & 100 & 0 & 50 & 0 \\
0 & 0 & 100 & 0 & 50 \\
50 & 0 & 0 & 100 & 0 \\
0 & 50 & 0 & 0 & 100
\end{array}\right)
$$


To choose one of the energy hub load demand pattern a binary switching vector $s_{a}$ is used. For example, to choose the first column, the first element of $s_{a}$ is set to binary integer 1, i.e., $s_{a}=\left[\begin{array}{lllll}1 & 0 & 0 & 0 & 0\end{array}\right]$. The energy hub load demand pattern for optimisation is given as:

$$
\begin{aligned}
L_{a}^{\top} & =X_{a}^{C} * s_{a}^{\top} \\
& =\left(\begin{array}{ccccc}
100 & 0 & 50 & 0 & 0 \\
0 & 100 & 0 & 50 & 0 \\
0 & 0 & 100 & 0 & 50 \\
50 & 0 & 0 & 100 & 0 \\
0 & 50 & 0 & 0 & 100
\end{array}\right)\left(\begin{array}{l}
1 \\
0 \\
0 \\
0 \\
0
\end{array}\right) \\
L_{a} & =\left[\begin{array}{lllll}
100 & 0 & 0 & 50 & 0
\end{array}\right]
\end{aligned}
$$

Similarly, to choose the second, third, fourth and fifth column of the circulant matrix $X_{a}^{C}$, the second, third, fourth, fifth element of $s_{a}$ is set to binary integer 1 respectively. If $\varepsilon_{a}^{t}$ is variable and bounded by a minimum hourly load level $\gamma_{a}^{\min } \geq 0$ and a maximum hourly load level $\gamma_{a}^{\max }$, with a positive constant $E_{a}$ denoting the total daily energy requirement limit for the $a^{\text {th }}$ energy hub load, the load demand scheduling plan $L_{a}$

where $L_{E T, t}=L_{E \text { fixed,a }}^{t}+L_{E \text { flexible, } a}^{t}+L_{\text {Enighttime, },}^{t}$, i.e, sum of fixed, flexible and night-time energy hub loads at any given time $t$. The general DR optimisation problem formulation for the whole day is given as:

$$
D R_{\text {min }}=\min \left[\sum_{t=1}^{T} \rho_{t} L_{E T, t}\right]
$$

\subsection{DR-DEED problem formulation}

The first step in the DR-DEED optimization model is to solve the demand response (DR) objective function. The objective of the DR strategy is to minimize the electricity cost of energy hub customers. The output load profile from the DR objective function is used as an input to the DEED dispatch model. Given a specific period of operation for the thermal power generation units and CHP units, the DEED dispatch model is about simultaneously minimizing both generation cost and the amount of emission; and at the same time ensuring that the system network load demand and generation units operation constraints are satisfied.

Assuming that the electricity pricing vectors for all the hours of the day are given as $\rho=\left(\rho_{1}, \rho_{2}, \ldots, \rho_{24}\right)$, the electricity cost at each hour $t, t \in 1,2, \ldots, T$ is given by:

$$
E H_{\text {cost }, t}=\rho_{t} * L_{E T, t}
$$

subject to: Flexible and non-flexible energy hub load constraints, i.e., Eqs 21-28 
The energy hub load demand $L_{E T, t}$ that minimizes the electricity cost for the whole day becomes the input to the DEED optimisation problem. In this stage, in order to simultaneously minimize both generation cost and amount of emission a weighting factor $\mu$ is introduced. The weighting factor is necessary to convert the multi-objective optimization problem into a weighted single objective optimization problem so that a balance between the two contradicting objective optimization problems is achieved. The weighting factor, $\mu$, blends the generation cost and the amount emission generation units. The mathematical formulation of the weighted single objective optimization can be expressed as:

$$
D R-D E E D=\min \left[\mu C_{c o s t}+(1-\mu) E_{\text {cost }}+D R_{\text {min }}\right]
$$

subject to: (i) Power and heat demand balance constraints (ii) CHP units and thermal power generation units constraints, i.e., Eqs 4- 9, 10-15, 19 and 20, (iii) Flexible and non-flexible energy hub load constraints, i.e., Eqs 21-28,

where:

$$
\begin{aligned}
C_{\text {cost }} & \left.=C_{i}^{G}\left(P_{i, t}\right)+C_{i}^{C H P}\left(P_{i, t}^{C H P}, H_{i, t}^{C H P}\right)\right] \\
E_{\text {cost }} & =E_{i}\left(P_{i, t}\right)
\end{aligned}
$$

The power and heat demand balance constraints is dependent on the scenario being considered. These constraints are therefore explained in each scenario considered. In this analysis, two stages were involved. The first stage investigated the effect of DR strategy on energy hub customers' electricity cost. Two scenarios were considered, i.e., (1) Without DR strategy (2) With DR strategy In the second stage, the power import between energy hubs, generation cost and amount of emission were investigated for 8 different scenarios. Scenario 2 of the first stage was used in the second stage for all the scenarios considered. In the second stage, 8 scenarios were considered in the case study and are given in the table below:

\begin{tabular}{ll}
\multicolumn{2}{c}{ Table 8: Possible combinations of communication between the energy hub system network } \\
\hline Scenario & Possible combination \\
\hline 1 & Distributed control scheme without communication \\
2 & Centralised control scheme \\
3 & Distributed control scheme with communication: $E 1 \rightarrow E 2 \rightarrow E 3$ \\
4 & Distributed control scheme with communication: $E 1 \rightarrow E 3 \rightarrow E 2$ \\
5 & Distributed control scheme with communication: $E 2 \rightarrow E 1 \rightarrow E 3$ \\
6 & Distributed control scheme with communication: $E 2 \rightarrow E 3 \rightarrow E 1$ \\
7 & Distributed control scheme with communication: $E 3 \rightarrow E 1 \rightarrow E 2$ \\
8 & Distributed control scheme with communication: $E 3 \rightarrow E 2 \rightarrow E 1$ \\
\hline
\end{tabular}

The arrow shows the sequence of communication between the energy hubs.

\subsection{Case 1: Effect of DR strategies}

In the first scenario, i.e., without DR strategy, flexible appliances are set to start at the beginning of their preference starting time. The electricity pricing vector of the respective energy hub customers are used in Eq. 30 to calculate their respective total electricity costs. In the case where the DR strategy is used, i.e., Scenario 2, the DR optimization model of Eq. 30 is used to minimize the total electricity cost of the 
respective energy hub customers. The minimization of the total electricity cost is achieved by changing the parameters on the power-shiftable and time-shiftable energy hub loads under their respective constraints. Table 9 below shows the different total electricity costs for the energy hubs. It can be clearly seen that when the DR strategy is used, the electricity cost of the energy hub customers is reduced. The results therefore show the significance of taking into account the load shifting capabilities of energy hub loads. Figures 7 -

Table 9: Energy hub customer electricity cost for different DR strategies

\begin{tabular}{lll}
\hline Energy Hub & With DR strategy & Without DR strategy \\
\hline 1 & $5.5761 \times 10^{5}$ & $5.828 \times 10^{5}$ \\
2 & $6.9376 \times 10^{5}$ & $6.9644 \times 10^{5}$ \\
3 & $9.5403 \times 10^{5}$ & $9.6948 \times 10^{5}$ \\
\hline
\end{tabular}

9 show the actual allocation of time for the individual energy hub loads. By scheduling the sequential time shiftable energy hub loads at their best times of operation and adjusting the power shiftable energy hub loads to their best level of operation, the total electricity cost of the energy hub customers were reduced according to their respective time varying electricity tariff pricing structure.

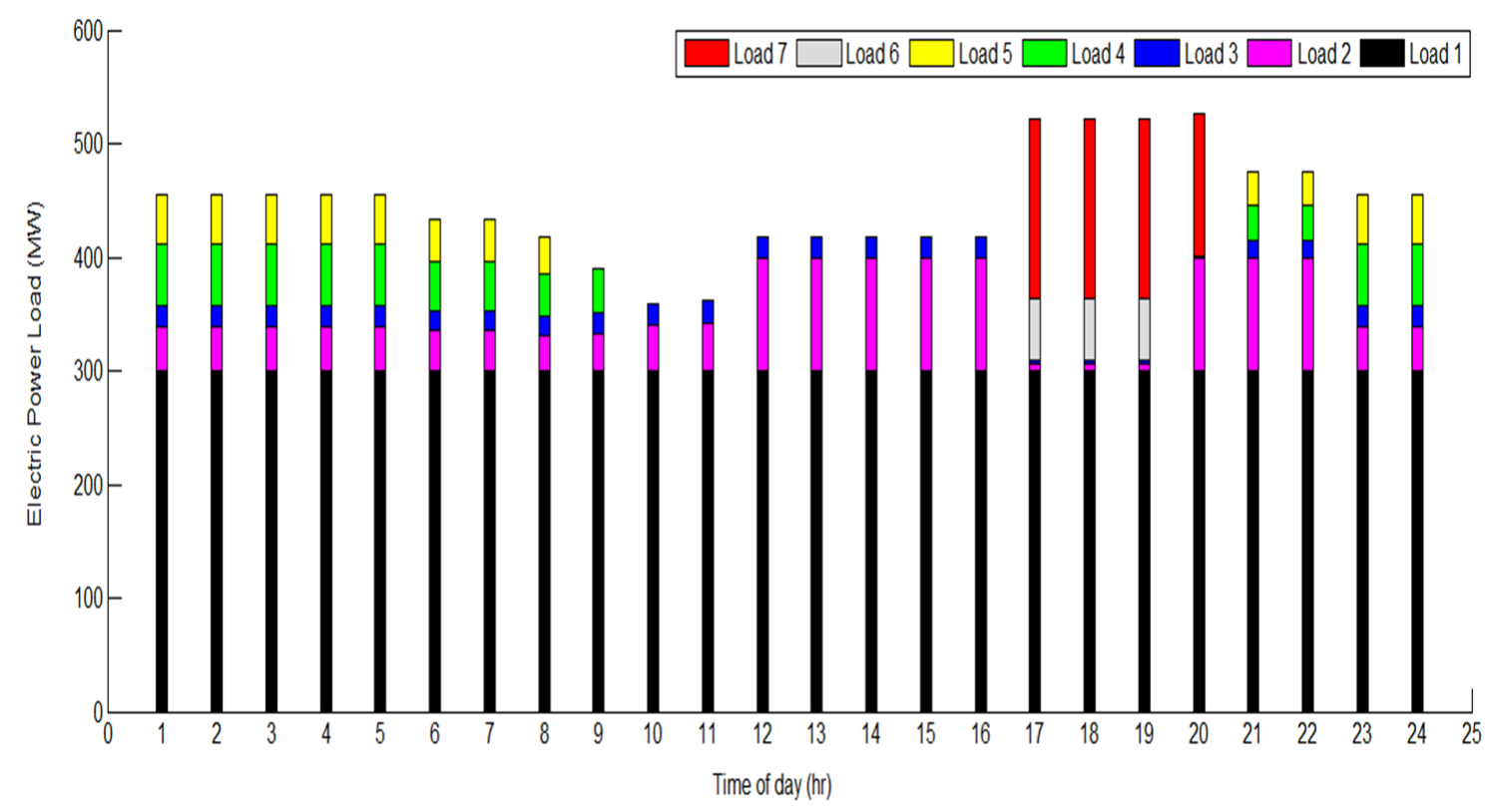

Figure 7: Residential customers optimal load for energy hub 1 


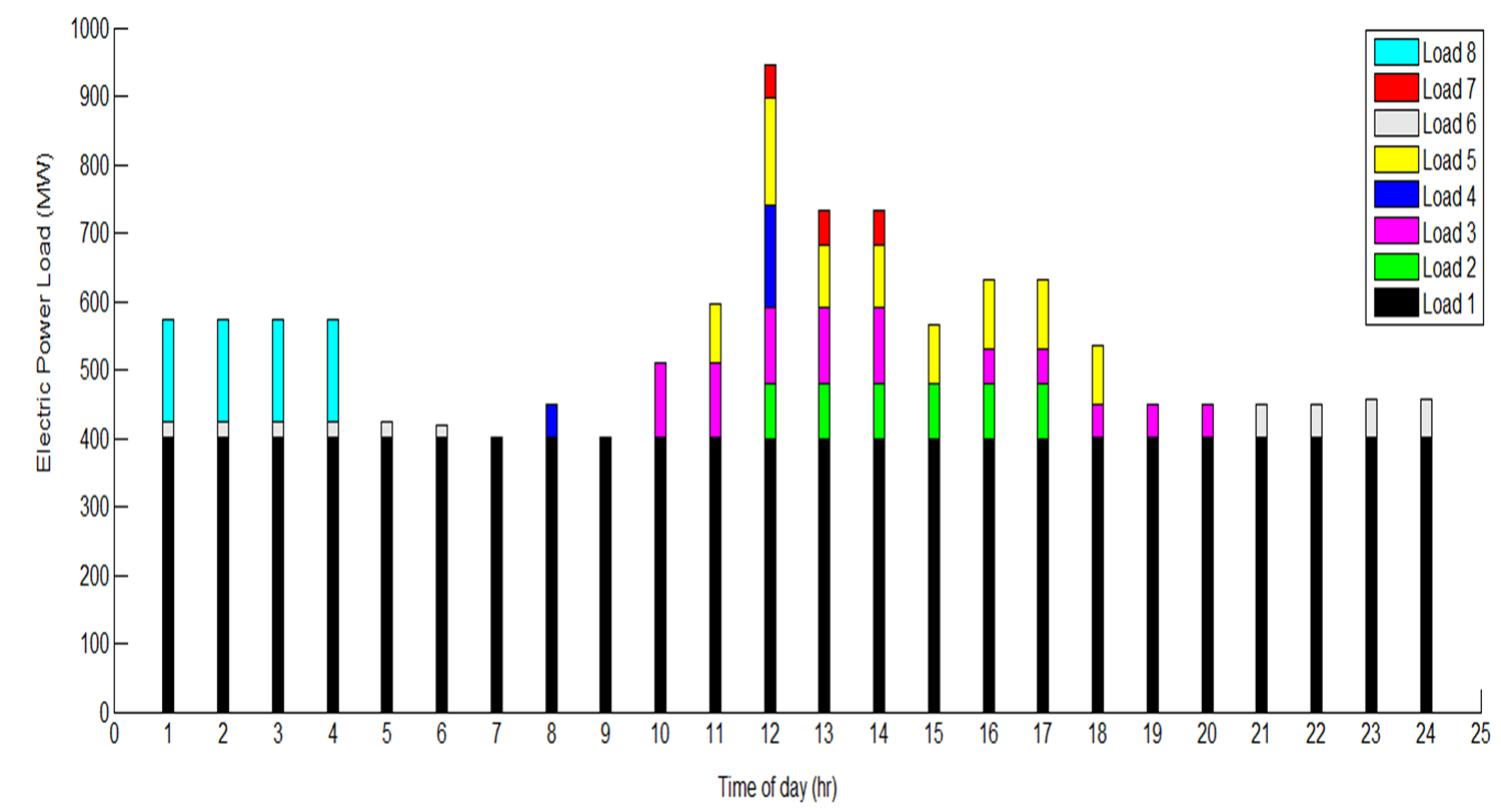

Figure 8: Commercial customers optimal load for energy hub 2

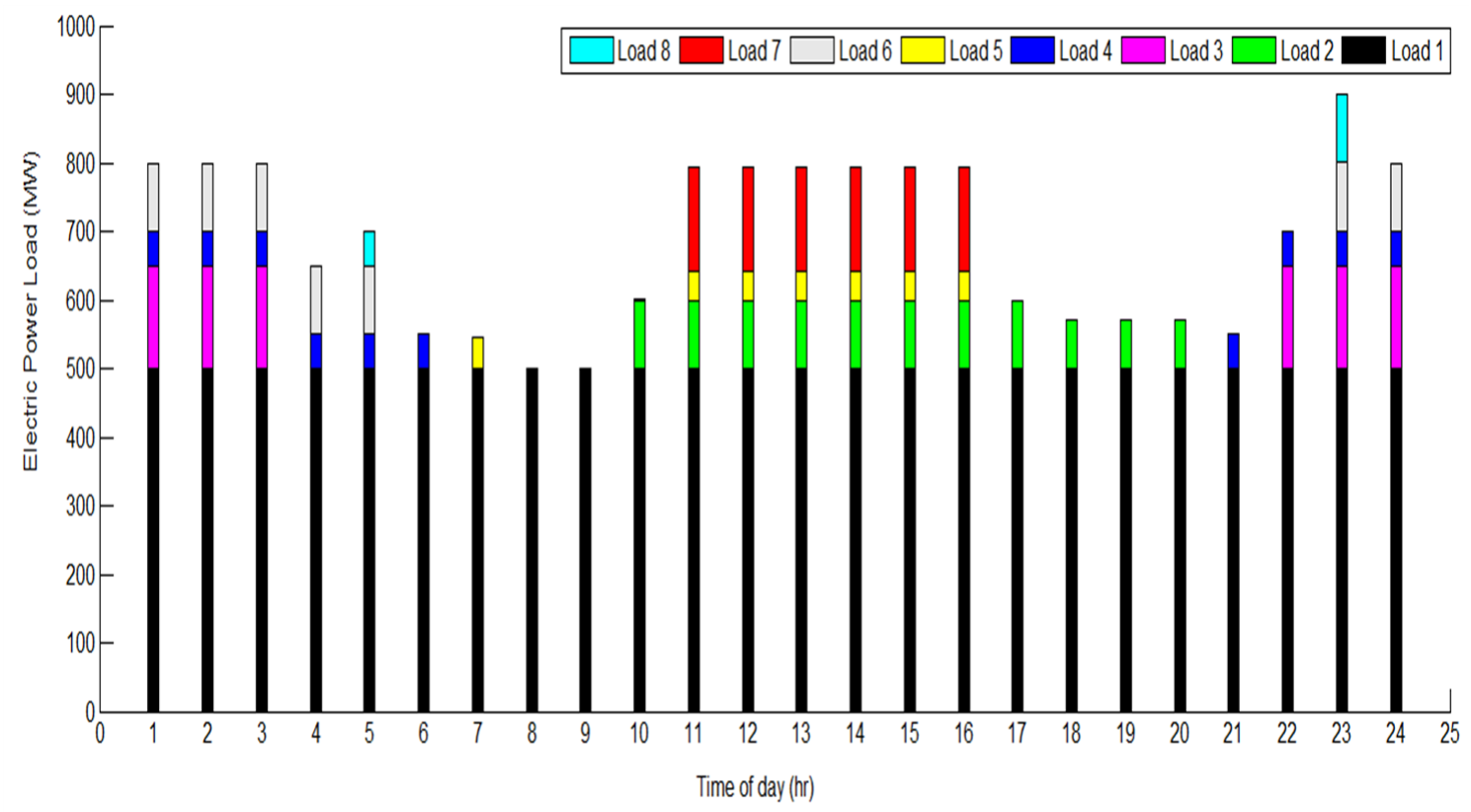

Figure 9: Industrial customers optimal load for energy hub 2

\subsection{Case 2: Effect of energy hub coordination}

\subsubsection{Scenario 1: Distributed control scheme without communication of energy hubs}

In this case, the energy hubs are optimized individually and there is no sharing of excess energy from each energy hub. 
The electricity pricing vector for the residential customers is used in Eq. 30. The output optimal energy hub load is then used to optimise the generation cost and amount of emission using Eq. 31. The power and heat demand balance for the energy hub is given by:

$$
L_{E T, t}=C_{T R} * G_{e i, t}+C_{P V} * G_{P V 1, t}
$$

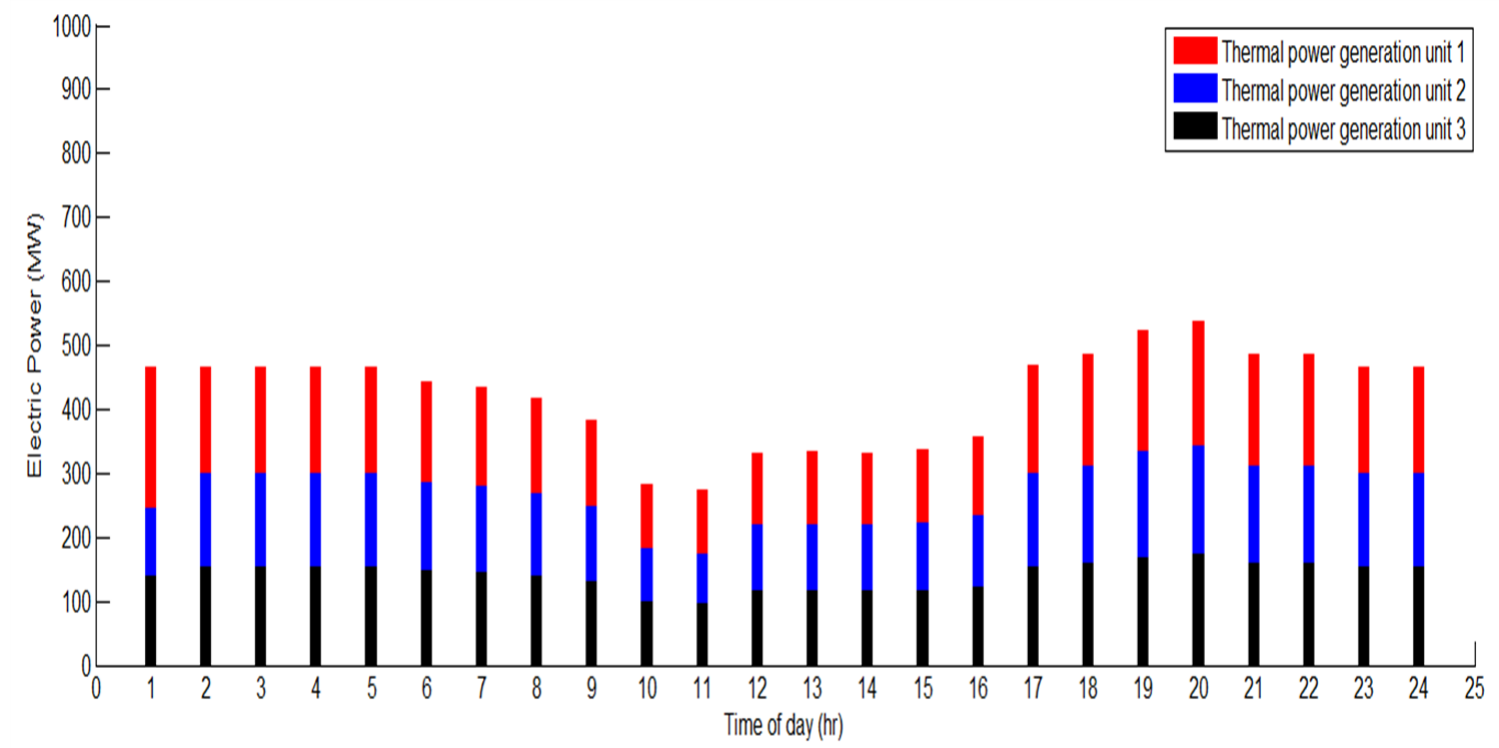

Figure 10: Optimal power generation output for thermal generation units at energy hub 1

\section{- Energy hub 2: Commercial customers}

Similarly, the electricity pricing vector for the commercial customers is used in Eq. 30 and the output optimal energy hub load become the input to Eq. 31. The power and heat demand balance for the energy hub is given as:

$$
\left[\begin{array}{c}
L_{E T, t} \\
L_{H T, t}
\end{array}\right]=\left[\begin{array}{ccc}
C_{T R} & C_{P V 2} & C_{n g E} \\
0 & 0 & C_{n g H}
\end{array}\right]\left[\begin{array}{c}
G_{e, t} \\
G_{P V 2, t} \\
G_{n g, t}
\end{array}\right]
$$




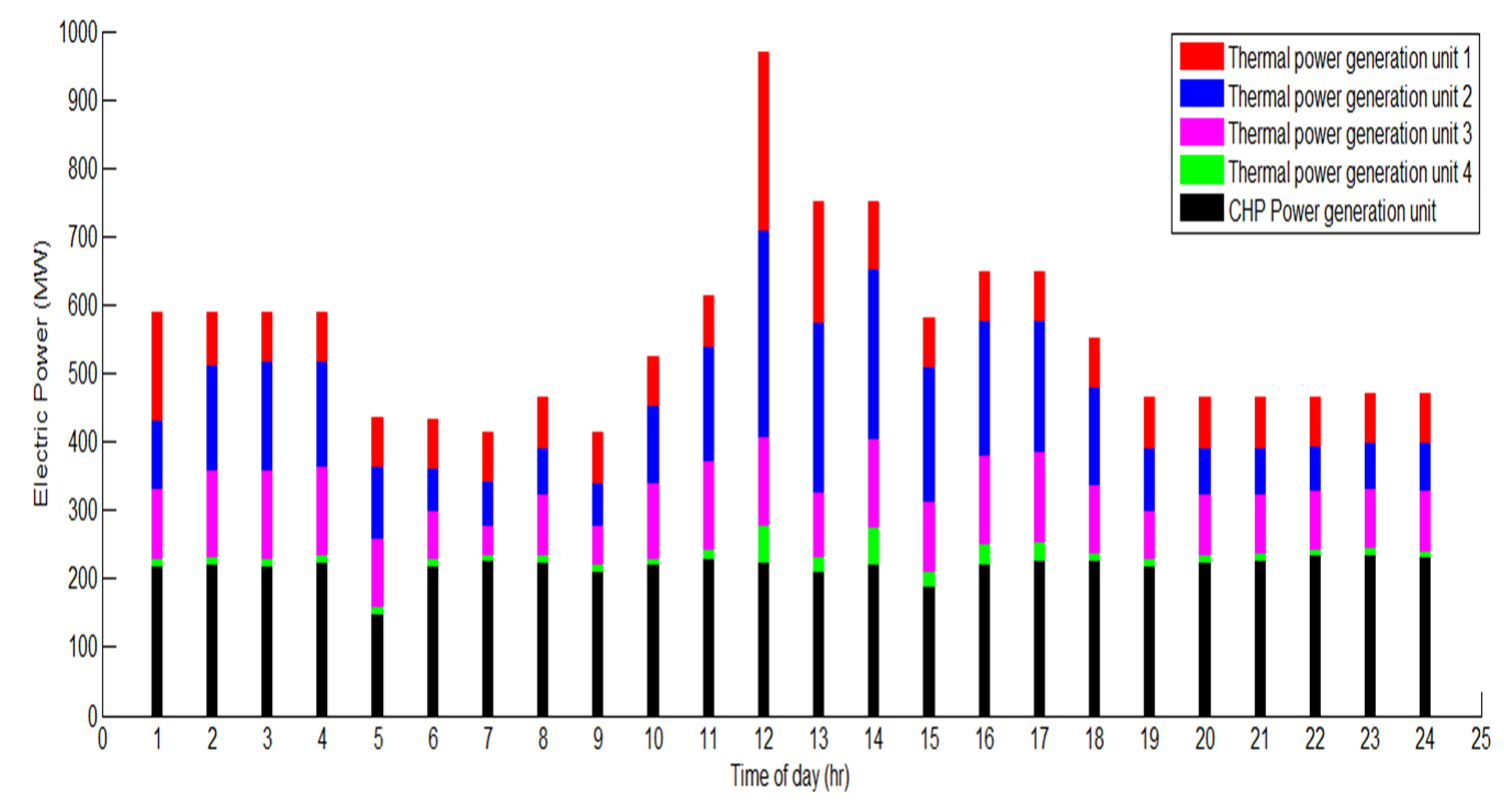

Figure 11: Optimal power generation output for thermal generation units at energy hub 2

\section{- Energy hub 3: Industrial customers}

The electricity pricing vector for the industrial customers is used in Eq. 30. The output optimal energy hub

load is then used in Eq. 31 to optimise the generation cost and amount of emission for the energy hub. The power and heat demand balance for the energy hub is given as:

$$
\left[\begin{array}{c}
L_{E T, t} \\
L_{H T, t}
\end{array}\right]=\left[\begin{array}{cc}
C_{T R} & C_{n g E} \\
0 & C_{n g H}
\end{array}\right]\left[\begin{array}{c}
G_{e, t} \\
G_{n g, t}
\end{array}\right]
$$

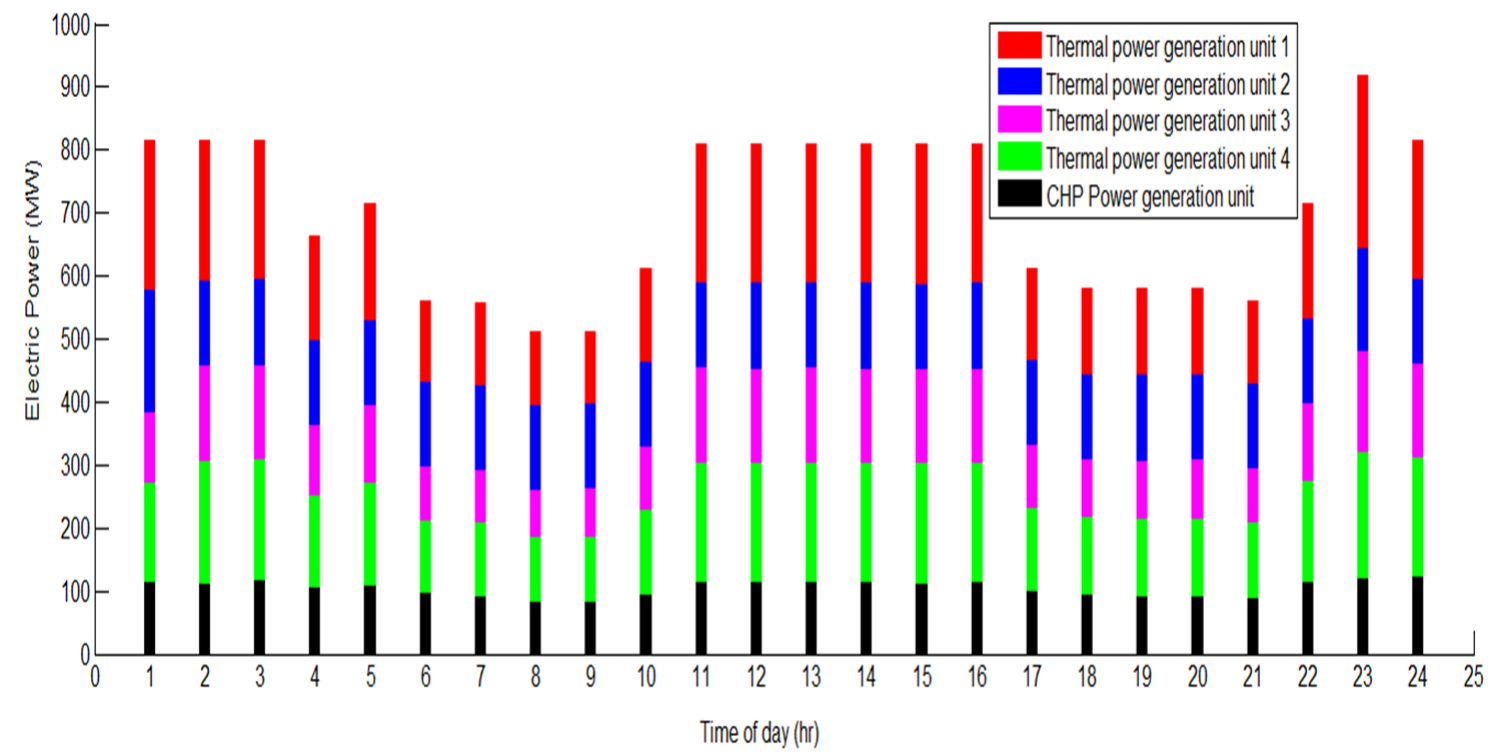

Figure 12: Optimal power generation output for thermal generation units at energy hub 3 


\subsubsection{Scenario 2: Centralised Control Scheme: DR-DEED Modelling}

In a centralised energy hub system network, the central supervisory controller receives initial objective energy hub load profile and electricity pricing as inputs from different energy hubs. It then set the required load control actions in order to minimize the cost of electricity to the respective energy hub customers using Eq. 30. The output load profile is used as an input to the DEED optimisation problem and is solved using Eq. 31. The power and heat demand balance constraints used in this analyses is explained below.

\section{- Power and heat demand balance constraints}

From the case study, the converter efficiency data for each converter is similar, i.e., $C_{T R 1}=C_{T R 2}=C_{T R 3}$, $C_{P V 1}=C_{P V 2}$ and $C_{N G 1}=C_{N G 2}$. The total power and heat demand balance for the whole system network is therefore given by:

$$
\begin{aligned}
L_{E T, t} & =C_{T R} \sum_{i=1}^{N} P_{i}^{t}+C_{n g E} \sum_{j=1}^{Z} P_{n g e, j}^{t}+C_{P V} \sum_{k=1}^{Y} P_{P V, k}^{t} \quad \forall t \in T \\
L_{H T, t} & =C_{n g H} \sum_{j=1}^{Z} G_{n g H, j}^{t} \quad \forall t \in T
\end{aligned}
$$

where N, Z, Y are the total number of thermal power generation units, CHP power generation units and solar PV power generation units in the energy hub network. $P_{i}^{t}$ is the electric power generation by $i^{t h}$ thermal power generation unit at any given time $t, P_{n g e, j}^{t}$ is the electric power generation by $j^{t h}$ CHP unit at any given time $t, G_{n g H, j}^{t}$ is the heat generation by $j^{t h} \mathrm{CHP}$ unit at any given time $t$ and $P_{P V, k}^{t}$ is the electric power generation by $k^{t h}$ solar PV power generation unit at any given time $t$.

\subsubsection{Distributed control scheme with communication: Scenario 3 - Scenario 8}

In this case, all the scenarios are solved in the same fashion. Each energy hub is able to communicate with neighbouring energy hubs in order to balance its power and heat demand. Excess energy from each energy hub is shared among neighbouring energy hubs. The exchange of information between the energy hubs enable the minimization of generation cost, amount of emission and electricity cost in all the energy hubs. There are six possible ways of communication between the three energy hubs. Each possible way of communication was considered as a scenario. DR-DEED optimisation was applied to all the possible scenarios and comparison were done in terms of the optimal power import by energy hubs, generation cost and amount of emission. The formulation of the problem uses equations that are derived from the individual energy hub optimisation problems of Scenario 1. However, virtual power plants are used to represent excess energy from each energy hub, which maybe passed on to the next energy hub depending on the possible combination followed. The DR-DEED optimisation for each scenario have the following steps:

- Step 1: Solve the DR optimisation model for the energy hub under consideration as in Scenario 1.

- Step 2: The output load from the energy hub is used to perform DEED optimisation for the respective energy hub 
Table 10: Optimal power output for thermal power generation units and CHP generation units of the multi-energy hub system

\begin{tabular}{|c|c|c|c|c|c|c|c|c|c|c|c|c|c|}
\hline & & \multicolumn{12}{|c|}{ Time $(h)$} \\
\hline & Gen. No & 1 & 2 & 3 & 4 & 5 & 6 & 7 & 8 & 9 & 10 & 11 & 12 \\
\hline \multirow{3}{*}{ Hub 1} & 1 & 220.00 & 167.06 & 159.96 & 136.98 & 121.65 & 100.00 & 100.00 & 100.00 & 100.00 & 100.00 & 132.43 & 199.04 \\
\hline & 2 & 107.48 & 146.39 & 140.59 & 121.79 & 109.27 & 86.81 & 81.19 & 79.07 & 63.93 & 85.38 & 118.08 & 172.53 \\
\hline & 3 & 140.00 & 153.07 & 148.05 & 131.80 & 120.97 & 101.55 & 96.69 & 94.85 & 81.76 & 100.31 & 128.59 & 175.67 \\
\hline \multirow{5}{*}{ Hub 2} & 1 & 160.00 & 80.00 & 73.00 & 73.00 & 73.00 & 73.00 & 73.00 & 73.00 & 73.00 & 73.00 & 73.00 & 73.00 \\
\hline & 2 & 190.00 & 240.00 & 285.46 & 253.89 & 232.85 & 195.13 & 185.69 & 182.13 & 156.70 & 192.72 & 247.65 & 300.00 \\
\hline & 3 & 110.00 & 130.00 & 130.00 & 130.00 & 130.00 & 130.00 & 130.00 & 130.00 & 130.00 & 130.00 & 130.00 & 130.00 \\
\hline & 4 & 47.74 & 55.00 & 55.00 & 55.00 & 49.28 & 29.86 & 25.00 & 23.17 & 10.08 & 28.62 & 55.00 & 55.00 \\
\hline & CHP 1 & 218.20 & 219.98 & 218.02 & 222.11 & 224.78 & 219.80 & 224.07 & 223.36 & 222.82 & 218.56 & 227.27 & 221.22 \\
\hline \multirow{7}{*}{ Hub 3} & 1 & 220.00 & 167.06 & 159.96 & 136.97 & 121.65 & 100.00 & 100.00 & 100.00 & 100.00 & 100.00 & 132.43 & 199.04 \\
\hline & 2 & 107.48 & 146.39 & 140.59 & 121.79 & 109.27 & 86.81 & 81.19 & 79.07 & 63.93 & 85.38 & 118.08 & 172.525 \\
\hline & 3 & 160.00 & 145.67 & 140.66 & 135.00 & 135.00 & 135.00 & 135.00 & 135.00 & 135.00 & 135.00 & 135.00 & 168.27 \\
\hline & 4 & 78.07 & 111.72 & 106.70 & 90.44 & 79.61 & 60.19 & 57.00 & 57.00 & 57.00 & 58.95 & 87.23 & 134.32 \\
\hline & CHP 2 & 111.80 & 110.74 & 115.13 & 111.05 & 110.44 & 121.17 & 117.24 & 115.28 & 113.77 & 113.31 & 112.56 & 112.11 \\
\hline & & \multicolumn{12}{|c|}{ Time $(h)$} \\
\hline & Gen. No & 13 & 14 & 15 & 16 & 17 & 18 & 19 & 20 & 21 & 22 & 23 & 24 \\
\hline \multirow{3}{*}{ Hub 1} & 1 & 163.79 & 163.03 & 139.11 & 152.17 & 138.21 & 121.51 & 113.28 & 116.69 & 106.16 & 126.31 & 154.76 & 139.43 \\
\hline & 2 & 143.72 & 143.09 & 123.54 & 134.22 & 122.81 & 109.16 & 102.43 & 105.21 & 96.61 & 113.08 & 136.34 & 123.81 \\
\hline & 3 & 150.76 & 150.22 & 133.31 & 142.54 & 132.68 & 120.87 & 115.05 & 117.46 & 110.02 & 124.26 & 144.38 & 133.54 \\
\hline \multirow{5}{*}{ Hub 2} & 1 & 73.00 & 73.00 & 73.00 & 73.00 & 73.00 & 73.00 & 73.00 & 73.00 & 73.00 & 73.00 & 73.00 & 73.00 \\
\hline & 2 & 290.72 & 289.67 & 256.83 & 274.76 & 255.60 & 232.67 & 221.36 & 226.04 & 211.59 & 239.25 & 278.32 & 257.27 \\
\hline & 3 & 130.00 & 130.00 & 130.00 & 130.00 & 130.00 & 130.00 & 130.00 & 130.00 & 130.00 & 130.00 & 130.00 & 130.00 \\
\hline & 4 & 55.00 & 55.00 & 55.00 & 55.00 & 55.00 & 49.19 & 43.37 & 45.78 & 38.34 & 52.58 & 55.00 & 55.00 \\
\hline & CHP 1 & 215.36 & 220.16 & 218.02 & 220.33 & 225.49 & 225.49 & 234.73 & 223.53 & 225.13 & 232.07 & 233.67 & 229.22 \\
\hline \multirow{5}{*}{ Hub 3} & 1 & 163.79 & 163.03 & 139.11 & 152.17 & 138.21 & 121.51 & 113.28 & 116.69 & 106.16 & 126.31 & 154.76 & 139.43 \\
\hline & 2 & 143.72 & 143.09 & 123.54 & 134.22 & 122.81 & 109.16 & 102.43 & 105.21 & 96.61 & 113.08 & 136.34 & 123.81 \\
\hline & 3 & 143.36 & 142.82 & 135.00 & 135.15 & 135.00 & 135.00 & 135.00 & 135.00 & 135.00 & 135.00 & 136.98 & 135.00 \\
\hline & 4 & 109.41 & 108.87 & 91.96 & 101.19 & 91.33 & 79.52 & 73.70 & 76.11 & 68.67 & 82.91 & 103.02 & 92.19 \\
\hline & CHP 2 & 112.71 & 111.80 & 111.65 & 111.80 & 111.50 & 115.28 & 113.31 & 114.22 & 115.28 & 118.00 & 119.36 & 121.63 \\
\hline
\end{tabular}


- Step 3: Calculate the excess energy for the energy hub

- Step 4: The excess energy from the respective energy hub is used as a virtual power plant in the corresponding energy hub that is being fed

- Step 5: Repeat Step 1 - Step 4 for the next energy hub including the virtual power plant (i.e. excess energy from first energy hub) to balance the power and heat demand of the respective energy hub

- Step 6: Repeat Step 1 - Step 4 for the third energy hub including the virtual power plant (i.e. excess energy from first and second energy hub) to balance the power and heat demand of the respective energy hub.
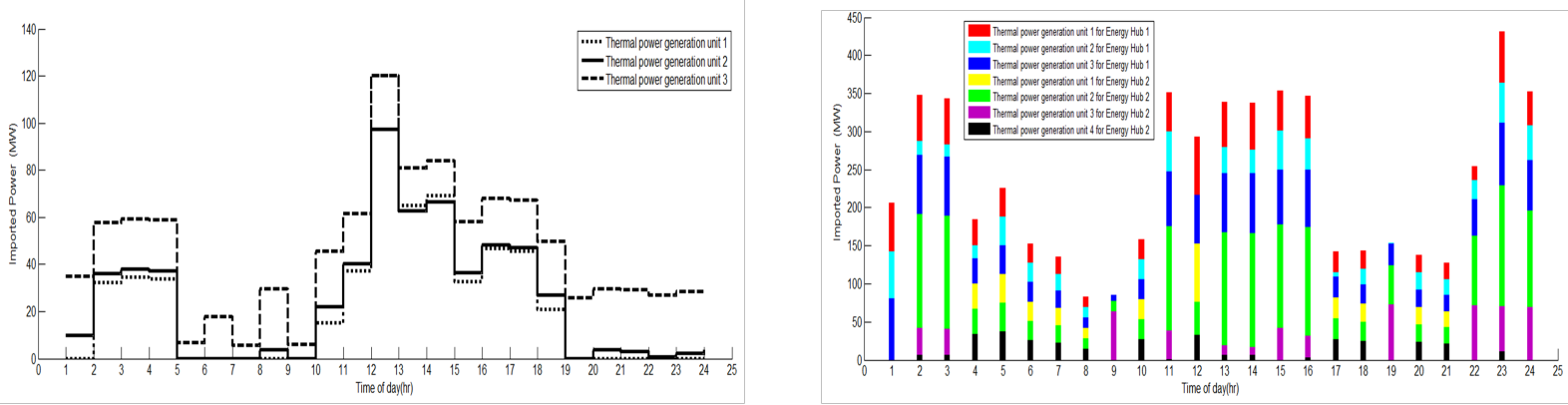

Figure 13: Optimal power import for thermal generation units for Scenario 3 between energy hub 1 to energy hub 2

Figure 14: Optimal power import for thermal generation units for Scenario 3 between energy $1 \& 2$ to energy hub 3
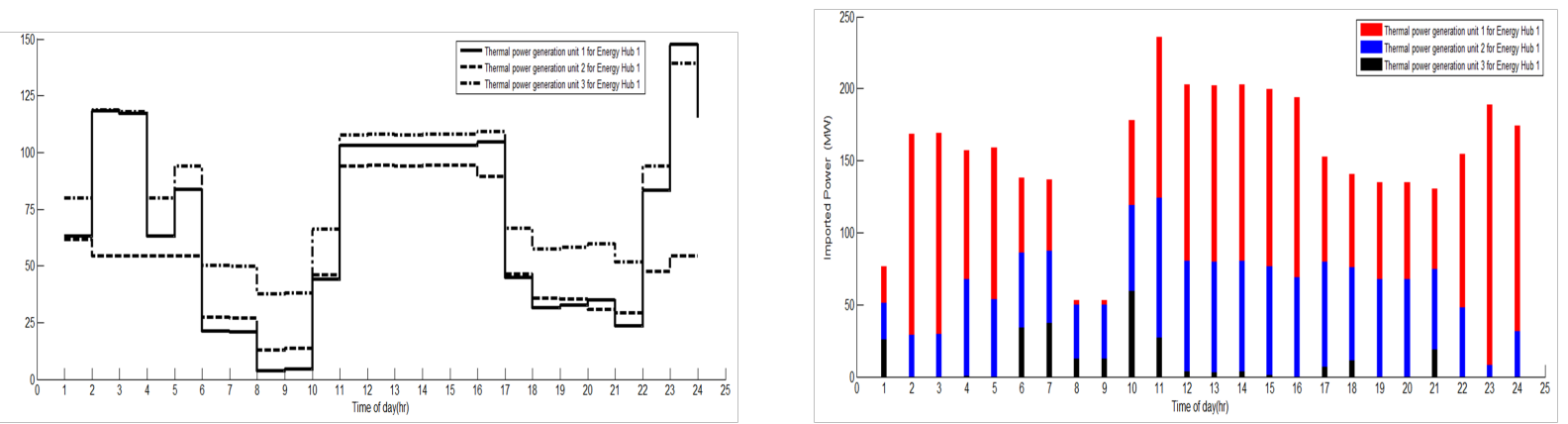

Figure 15: Optimal power import for thermal generation units for Scenario 4 between energy hub 1 to energy hub 3

Figure 16: Optimal power import for thermal generation units for Scenario 4 between energy hub $1 \& 3$ to energy hub 2

\section{Discussion of results}

Figure 25 shows the amount of emission from generation units for all the scenarios considered in Case 2. Generally, the weighting factor $\mu$ affects the amount of emission from all generation units for all the scenarios considered. On average, lowest amount of emission is achieved when Scenario 5 is employed. Giving more emphasis to the amount of emission of generation units, i.e., $\mu=0$, the centralised control scheme has the highest amount of emission while Scenario 5 has the lowest. In general, it can also be clearly seen from the figure that as the value of $\mu$ increases the amount of emission also increases with the exception of Scenario 


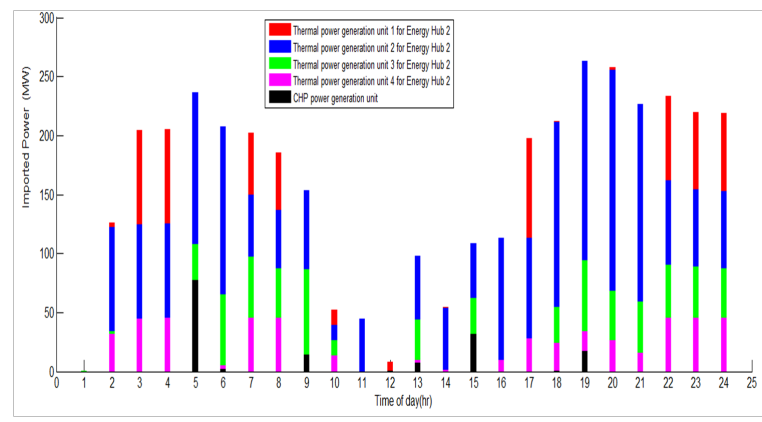

Figure 17: Optimal power import for thermal generation units for Scenario 5 between energy hub 2 to energy hub 1

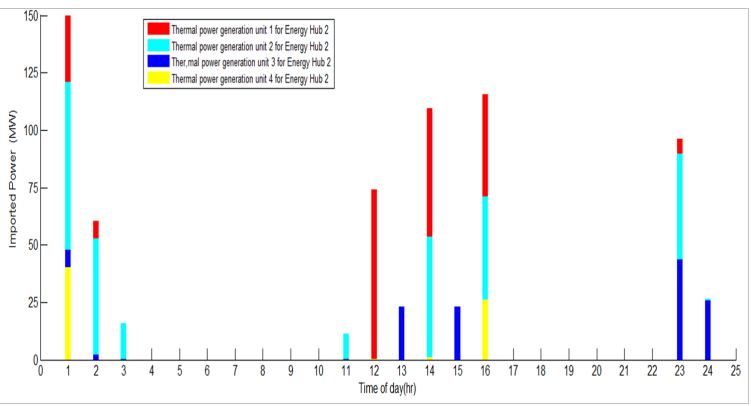

Figure 19: Optimal power import for thermal generation units for Scenario 6 between energy hub 2 to energy hub 3

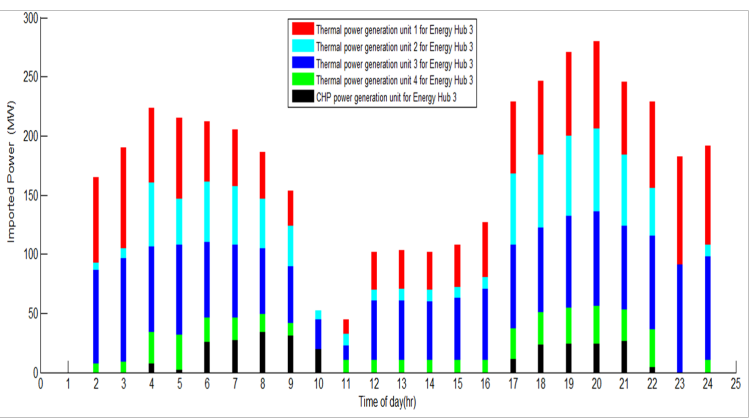

Figure 21: Optimal power import for thermal generation units for Scenario 7 between energy hub 3 to energy hub 1

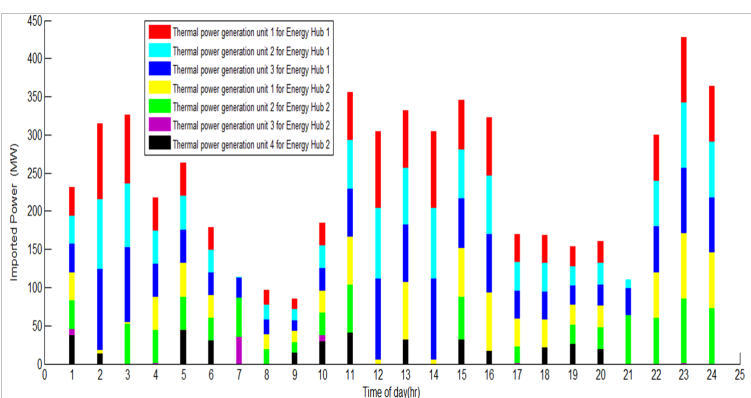

Figure 18: Optimal power import for thermal generation units for Scenario 5 between energy hub $2 \& 1$ to energy hub 3

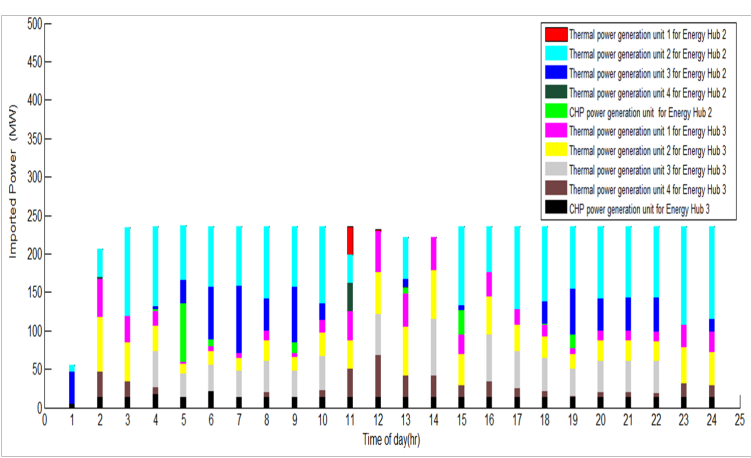

Figure 20: Optimal power import for thermal generation units for Scenario 6 between energy hub $2 \& 3$ to energy hub 1

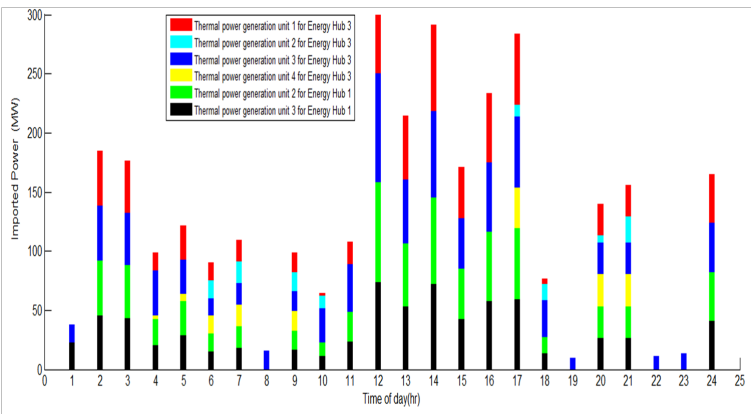

Figure 22: Optimal power import for thermal generation units for Scenario 7 between energy hub $3 \& 1$ to energy hub 2

4. There is a sharp increase of $\approx 250 \%$ in the amount of emission when $\mu$ is increased between 0 to 0.5 for Scenario 6. In Scenario 4, a reduction of less than $10 \%$ in the amount of emission is achieved when $\mu$ is increased from 0.5 to 1. For all the scenarios, the highest amount of emission is achieved in Scenario 6 when $\mu$ is set at either 0.5 or 1 . Scenario 1 and 2 are almost similar for all values of $\mu$. Scenario 2 has higher amount of emission for all values of $\mu$ when compared to Scenario 3, 4 and 5. This clearly shows that the amount of emission can be reduced with the use of networking between different energy hubs.

In the case of generation cost, i.e., Figure 26, the value of the weighting factor $\mu$ does not affect the generation cost. However, the coordination of the energy hubs have a great effect on the generation cost. The lowest generation cost is achieved when Scenario 1, i.e., distributed control scheme without communication 

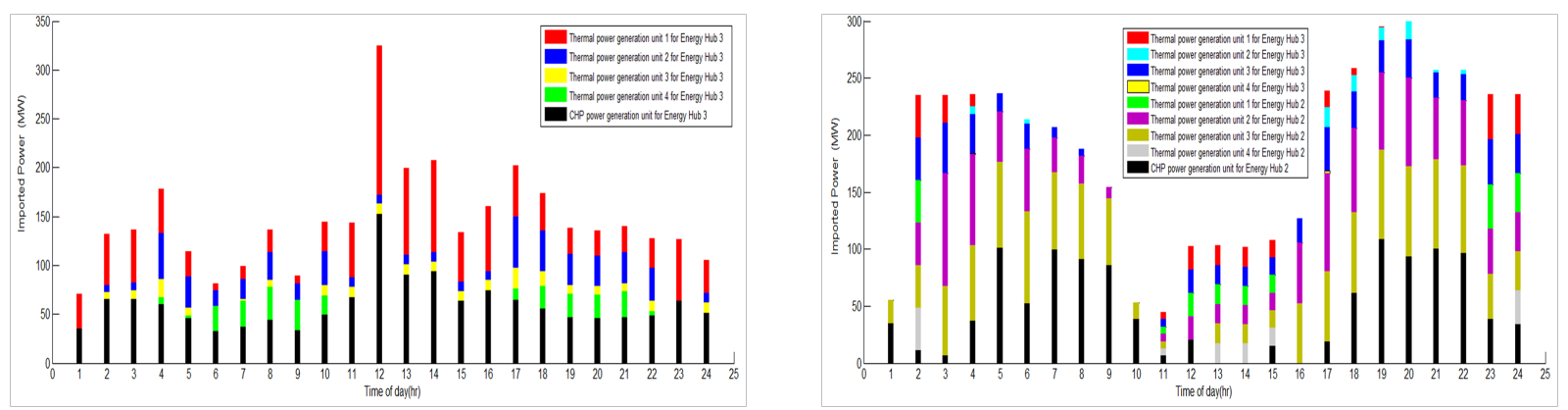

Figure 23: Optimal power import for thermal generation units for Scenario 8 between energy hub 3 to energy hub 2

Figure 24: Optimal power import for thermal generation units for Scenario 8 between energy hub $3 \& 2$ to energy hub 1

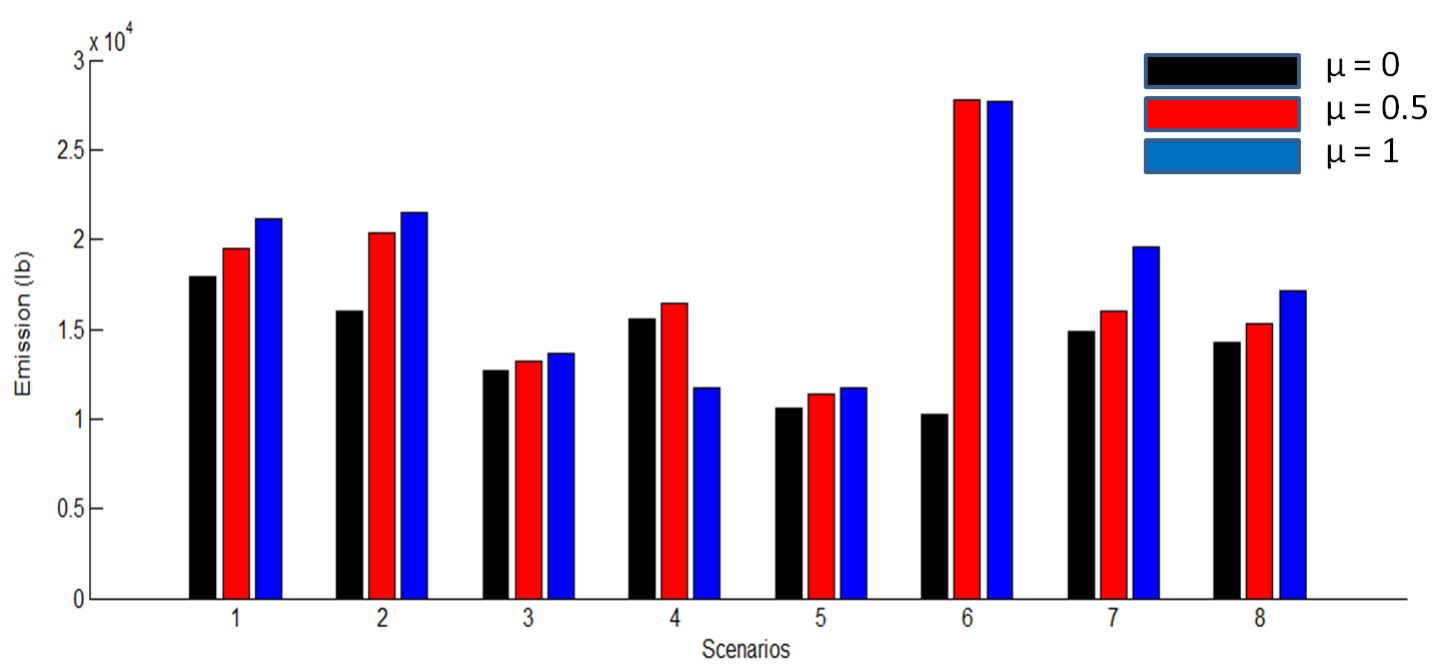

Figure 25: Amount of Emission from thermal power generation units for all scenarios

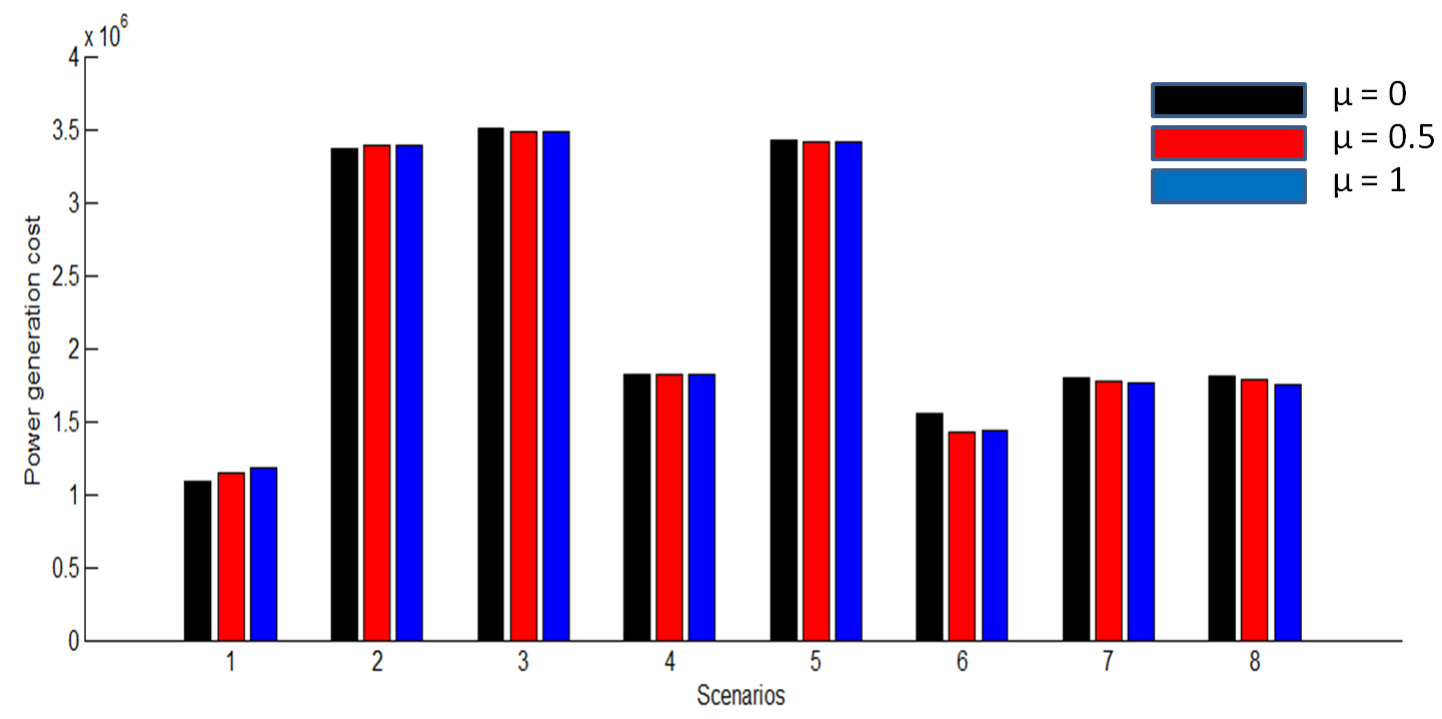

Figure 26: Generation cost of thermal power generation units for all scenarios

is employed. Scenario 4, 6, 7 and 8 also generally have low generation costs. The highest generation costs are achieved in Scenario 2, 3 and 5. The generation costs decrease by more than $50 \%$ in Scenarios 1, 4, 6, 7 and 
8 with respect to Scenarios 2, 3 and 5 . It can also be clearly seen that it is difficult to have a set of control schemes that gives both low amount of emission and generation costs at the same time. By decreasing the weighting factor $(\mu)$ or increasing the importance of emission, less amount of emission is generated at a higher generation cost for Scenario 2, 3 and 5. Whereas, increasing more emphasis on generation cost or increasing the weighting factor $(\mu)$, high amount of emission is generated at lower generation cost for Scenario 1, 6 , 7 and 8. When there is a balance between the amount of emission and the generation costs, i.e., $\mu=0.5$, the conditions generally favours distributed control strategies, (i.e., Scenario 4, 7 and 8) demonstrating the benefits of networking between energy hubs. There is therefore a need for a trade-off between generation costs and amount of emission when distributed control schemes are employed for the different sparsely located distributed energy hubs.

The value of power import from each energy hub where calculated with $\mu=0.5$. Generally, there are similarities in all the scenarios in terms of the power import profile from neighbouring energy hubs. The lowest power import is experience in Scenario 6 between energy hub 2 and 3. For most part of the day there is no import of power from energy hub 2. The maximum import of power is experienced at 0100 hours. When all energy hubs are treated as stand alone, i.e., Scenario 1, the power demand from all the thermal power generation units and CHP units generally follow the energy hub load demand profile. For energy hub 2 and 3, it can be clearly seen that the CHP units of the respective energy hubs provides almost their maximum power generation for all the 24 hours considered. This demonstrates that in order to make distributed energy systems like CHP more efficient, they should be used in the network of energy hubs.

In Scenario 4, the amount of power imported by energy hub 3 is much higher than that which is imported by energy hub 2 . The reason is that the thermal power generation units at energy hub 3 are too expensive to generate the power for the respective energy hub load. The power generation from CHP unit of energy hub 3 is exported to energy hub 1, contributing almost $13 \%$ of the total energy demand during peak hour at 2300 hours. The maximum power import by energy hub 2 and 3 occurs at 2300 hours and 1100 hours respectively. No CHP power is exported from energy hub 3 to energy hub 2.

In Scenario 7 all thermal power generation units export power to energy hub 2 throughout the whole day. Maximum power export is at 1200 hours and during this time the CHP unit of energy hub 3 and thermal power generation unit 1 have maximum power export. It is thus expected as this is the time when there is maximum energy hub load demand for energy hub 2. In the same scenario, power import from energy hub 1 attain a maximum of $\approx 300 \mathrm{MW}$ at 2000 hours compared to $\approx 700 \mathrm{MW}$ at 1200 hours for energy hub 2. There is therefore more power import from energy hub 2 than energy hub 1 . The minimum power import for energy hub 1 occurs at 0100 hours and 1000 hours - 1100 hours. The CHP unit for energy hub 2 supplies its maximum power to energy hub 1 at 1900 hours - 2200 hours and 0500 hours - 0900 hours. This demonstrates that for networks of energy hubs, there is a need for interactions or interdependency of energy hubs in order to achieve greatest benefits.

Table 10 shows optimised power output for thermal power generation units and CHP units of the multienergy hub system using the centralised control scheme. It is worthwhile to note that, both CHP units have their power output approximately following the heat demand. Peak power output demand is $\approx 234 \mathrm{~kW}$ and 
$\approx 122 \mathrm{~kW}$ for CHP 1 and CHP 2 respectively. The reason for this is that since the power generation of each CHP unit is dependent on its respective heat generation at any particular given time, the energy hub heat demand reaches its peak at these respective hours.

In Scenario 8, all generation units at energy hub 3 exported power to energy hub 2. This means that most of the thermal power generation units at hub 3 generate much cheaper power than those at energy hub 2. However, when both energy hubs are able to export to energy hub 1, it can be seen that an average of $\approx 250 \mathrm{MW}$ is exported to energy hub 1 throughout the whole day with the exception of a minimum of $\approx 50 \mathrm{MW}$ at 0100 hours. This points out that there is great advantage of controlling each energy hub and exporting the excess power to the neighbouring energy hubs. The excess energy can be exported using a different tariff strategy as a result of the transmission cost involved. In this research paper, however, export cost of excess energy from each energy hub is not included in the analyses. It should therefore be noted that the use of these other factors may change the results of the case study.

From the analyses of results above, it can be concluded that significant clean power generation, such as CHP, is needed in the network to achieve all of the benefits of networking. The results also show that policy makers, power system planners and operators have to give priority in balancing emission, generation costs and power export capabilities of energy hubs when designing control strategies for different sparsely located distributed energy hubs.

\section{Conclusion}

The case study presented in this paper shows the potential of DR strategies and energy hub coordination. The DR-DEED optimisation model applied in the case study allows for a comparison of more sustainable energy hub control schemes, including the evaluation of economic, environmental criteria and power import between energy hubs. Results show that the weighting factor $\mu$ have no significant relationship to the total generation cost of the power generation units in the energy hubs for all scenarios considered. However, a significant reduction of generation cost is achieved when different control strategies are employed for the energy hubs. The import of power from each energy hub in different scenarios is influenced by the generation cost of the energy hubs that are involved. Thermal power generation units with less generation cost export more excess power to neighbouring energy hubs than those that are costly. The fact that it is difficult to have a set of distributed control schemes that gives both low amount of emission and generation costs at the same time points for the need to accurately assess generation cost and amount of emission for all energy sources to come up with accurate multi-energy hub system management frameworks that are sustainable and environmentally friendly.

The focus of this contribution lies on the re-design of the urban energy systems. However, the design of an adequate control approach for such energy systems is not a trivial task, taking the different levels of centralized technologies and integrated technologies into account. This is not taken into account in the present contribution, and bears an additional area of research which has to be explored in the future. Further limitation are given due to omission of energy hub excess energy sells to neighbouring energy hubs, 
this simplification needs to be further explored. The final goal is to apply the developed procedures to larger energy systems with more than three hubs.

\section{References}

\section{References}

[1] S. Perry, J. Kleme, I. Bulatov, Integrating waste and renewable energy to reduce the carbon footprint of locally integrated energy sectors., Energy 33 (10) (2008) $1489-97$.

[2] M. Akorede, H. Hizam, E. Rouresmaeil, Distributed energy resources and benefits to the environment, Renewable and Sustainable Energy Reviews 14 (2010) $724-34$.

[3] H. Farhangi, A road map to integration: perspectives on smart grid development, IEEE Power Energy Magazine 12 (3) (2014) $52-66$.

[4] P. Mancarella, MES (Multi-Energy Systems): An overview of concepts and evaluation models, Energy $65(2014) 1-17$.

[5] M. Geidl, G. Koeppel, P. Favre-Perrod, B. Klockl, G. Andresson, K. Frolich, Energy hubs for the future, IEEE Power Energy Magazine 5 (1) (2007) 24 - 30.

[6] G. Chicco, P. Mancarella, Distributed multi-generation: A comprehensive view, Renew Sustainable Energy Reviews 13 (3) (2009) $335-51$.

[7] H. Ren, W. Gao, A MILP model for integrated plan and evaluation of distributed energy systems, Applied Energy 87 (3) (2010) $1001-14$.

[8] C. Weber, N. Shah, Optimisation based design of a district energy system for an eco-town in the United Kingdom, Energy 36 (2) (2011) 1292 - 308.

[9] ICF International. 2012 - The Future of Natural Gas in Mozambique: Towards a Natural Gas Master Plan., Consultant report for World Bank and Government of Mozambique.

[10] I. Gqada, Mozambiques Gas: An opportunity for South Africa?, SAIIA: Governance of Africas Resources Programme: POLICY BRIEFING 53 (2012) 1 - 3: http://www.saiia.org.za/policy-briefings/179mozambique-s-gas-an-opportunity-for-south-africa/file.

[11] A. Omu, R. Choudhary, A. Boies, Distributed energy resource system optimisation using mixed integer linear programming, Energy Policy 61 (2013) $249-66$.

[12] M. Nazar, M. Haghifam, Multiobjective electric distribution system expansion planning using hybrid energy hub concept., Electrical Power Systems Research 79 (6) (2009) 899 - 911.

[13] M. Arnold, R. Negenborn, G. Andersson, B. De Schutter, Distributed predictive control for energy hub coordination in coupled electricity and gas networks, Intelligent Infrastructure, Intelligent Systems, Control Automation: Science Engineering 42 (2010) 235 - 73. 
[14] J. Yoona, R. Bladicka, A. Novoselac, Demand response for residential buildings based on dynamic price of electricity, Energy Buildings 80 (2014) 531541.

[15] R. Evins, K. Orehounig, V. Dorer, J. Carmeliet, New formulations of the energy hub model to address operational constraints., Energy 73 (2014) $387-98$.

[16] A. Parisio, V. Carmen Del, A. Vaccaro, A robust optimization approach to energy hub management, International Journal Electrical Power Energy Systems 42 (1) (2012) 98 - 104.

[17] M. Schulze, L. Friedrich, M. Gautschi, Modeling and optimization of renewables: applying the energy hub approach, Sustainable Energy Technologies: IEEE International Conference on ICSET.

[18] J. Keirstead, M. Jennings, A. Sivakumar, A review of urban energy system models: Approaches, challenges and opportunities., Renewable and Sustainable Energy Reviews 16 (2012) 3847 -66.

[19] A. J. Conejo, J. M. Morales, L. Baringo, Real-time demand response model, IEEE Transaction on Smart Grid 3 (3) (2010) $236-242$.

[20] S. An, Q. Li, T. W. Gedra, Natural gas and electricity optimal power flow, IEEE PES Transmission and Distribution Conference (2003) 138 - 143.

[21] A. M. Elaiw, A. M. Shehata, M. A. Alghamdi, A model predictive control approach to Combined Heat and Power dynamic economic dispatch problem, Arab Journal of Science Engineering 39 (2014) 7117 7125.

[22] N. Nwulu, X. Xia, Multi-objective dynamic economic emission dispatch of electric power generation integrated with game theory based demand response programs, Energy Conversion and Management 89 (2015) $963-974$. 